\title{
INTERACCIONES, TOQUES EN PANTALLA Y APRENDIZAJE DE CUADRILÁTEROS
}

\author{
INTERACTIONS, TOUCHES ON SCREEN AND LEARNING OF QUADRILATERALS \\ INTERAÇÕES, TOQUES EM TELA E APRENDIZAGEM DE QUADRILÁTEROS
}

\author{
Marcelo Bairral \\ Universidad Federal Rural de Río de Janeiro, Río de Janeiro, Brasil \\ Recibido: 28/04/2021 - Aceptado: 08/06/2021 - Publicado: 01/12/2021 \\ Remita cualquier duda sobre esta obra a: Marcelo Bairral. \\ Email: mbairral@ufrrj.br
}

\begin{abstract}
RESUMEN
Además de la movilidad, ubicuidad y convergencia de medios de comunicación, las tabletas y smartphones permiten toques en pantalla. Estos toques pasan a integrar el sistema lingüístico y, consecuentemente, de pensamiento. Los dispositivos móviles pueden contribuir con los procesos de enseñanza y aprendizaje. Este artículo ilustra respuestas de estudiantes y futuros profesores y suscita, a partir de ellas, reflexiones sobre hallazgos geométricos producidos por estudiantes de Educación Media y de la Licenciatura en Matemática. Las actividades se orientaron hacia el aprendizaje de cuadriláteros con enfoque en la identificación, conceptualización, análisis de propiedades y producción de demostraciones, usando ambientes de geometría dinámica con toques en pantalla $(\mathrm{AGDcT})$. Los datos se produjeron a partir de las respuestas escritas a las tareas, diarios de los investigadores, observaciones y conversaciones a lo largo de las clases, construcciones en pantalla y videograbaciones. Acciones como desplazar, mover y aumentar se evidenciaron en el análisis y pueden enriquecer el repertorio de los sujetos cuando lidian con AGDcT. En la producción de justificaciones y demostraciones matemáticas, se evidenciaron dos dominios de manipulación en pantalla. Los toques en pantalla deben ser vistos como una forma más de cognición corporificada.
\end{abstract}

Palabras clave: Cuadriláteros; Toques en pantalla; Dominios constructivo y relacional; Justificaciones; Demostraciones.

\begin{abstract}
Beyond mobility, ubiquity and the convergence of media, tablets and smartphones allow touch screens. These touches become part of the linguistic system and, therefore, of thought. Mobile devices can contribute to the instruction and learning processes. The article illustrates answers from students and prospective teachers and raises, from them, reflections on geometric discoveries produced by high school and prospective teachers in mathematics. The activities aimed at learning quadrilaterals with a focus on identification, conceptualization, analysis of properties and the production of proofs, using dynamic geometric environment with touchscreen
\end{abstract}


(DGEwT). The data were produced in written responses to the tasks, researchers' diaries, observations and conversations throughout the classes, screen constructions and video recordings. Actions such as moving and increasing were evidenced in the analysis and can enrich the subjects' repertoire when dealing with AGDcT. Two domains of handling on screen are evidenced in the production of justifications and mathematical proofs. Screen touches should be seen as another form of embodied cognition.

Keywords: Quadrilaterals; Touches on screen; Constructive and relational realms; Justifications; Proofs.

\section{RESUMO}

Além da mobilidade, da ubiquidade e da convergência de mídias, tablets e smartphones permitem os toques em tela. Esses toques passam a integrar o sistema linguístico e, por conseguinte, de pensamento. Dispositivos móveis podem contribuir com os processos de ensino e de aprendizagem. $\mathrm{O}$ artigo ilustra respostas de alunos e licenciandos e suscita, a partir delas, reflexões sobre descobertas geométricas produzidas por estudantes do Ensino Médio e da Licenciatura em Matemática. As atividades visavam ao aprendizado de quadriláteros com foco na identificação, na conceituação, na análise de propriedades e na produção de provas, usando ambientes de geometria dinâmica com toques em tela (AGDcT). Os dados foram produzidos a partir das respostas escritas para as tarefas, diários dos pesquisadores, observações e conversas ao longo das aulas, construçóes em tela e videogravaçôes. Açôes como mexer, mover e aumentar foram evidenciadas na análise e podem enriquecer o repertório dos sujeitos quando lidam com AGDcT. Dois domínios de manipulação em tela são evidenciados na produção de justificativas e provas matemáticas. Toques em tela devem ser vistos como mais uma forma de cognição corporificada.

Palavras-chave: Quadriláteros; Toques em tela; Domínios construtivo e relacional; Justificativas; Provas.

\section{INTRODUCCIÓN}

A diferencia de los recursos de construcción geométrica (regla, compás, transportador, etc.) e, incluso, de softwares educativos, los ambientes de geometría dinámica con toques en pantalla (AGDcT) están provocando cambios en los procesos de enseñanza y aprendizaje. Por estar presentes en las manos de la mayoría de los individuos, estas tecnologías prescinden de laboratorios de informática, permiten compartir aplicaciones y no requieren de conexiones a Internet. De esta forma, estos ambientes pueden ampliar las posibilidades de enseñar y despertar el interés en el aprendizaje matemático.

Aunque en el escenario brasileño ya existe una agenda de investigación sobre dispositivos móviles con toques en pantalla (DMcTT, por sus siglas en portugués), la cual analiza cambios de naturaleza epistemológica (Assis, 2020; Assis \& Bairral, 2019), y/o cognitiva y didáctica (Bairral \& Carvalho, 2019; Bairral \& Henrique, 2021), en el escenario internacional también encontramos estudios enfocados en implicaciones epistemológicas (Arzarello et al., 2014; Sinclair \& Freitas, 2014). No obstante, aún predomina una mirada más didáctica o de naturaleza cognitiva (Calder et al., 2018). En lo didáctico se incluyen miradas atentas a la forma en que el profesor establece comunicaciones con sus alumnos cuando los dispositivos móviles entran en escena (Chao et al., 2016). 
Este artículo sintetiza e integra resultados ${ }^{1}$ de publicaciones anteriores, vinculadas al proyecto Construyendo y analizando prácticas educativas en educación matemática con dispositivos touchscreen, financiado por el CNPq (Brasil) y desarrollado en el marco del Grupo de Estudios e Investigaciones de las Tecnologías de la Información y Comunicación en Educación Matemática (Gepeticem, por sus siglas en portugués)2.

Los tres estudios tienen a los cuadriláteros como contenido central, el cual es abordado en los currículos de geometría de Brasil, sobre todo, con alumnos entre 12 y 15 años. La Tabla 1 resume aspectos de cada estudio que aquí será integrado. Para mayores detalles de cada uno, sugiero la lectura de las referencias indicadas ${ }^{3}$.

\section{Tabla 1}

Sintesis de los estudios ilustrados

\begin{tabular}{|c|c|c|c|c|c|}
\hline No. & Referencia(s) & Enfoque(s) & Participantes & $\begin{array}{c}\text { Dispositivo } \\
\text { utilizado } \\
\end{array}$ & $\begin{array}{c}\text { Principal(es) } \\
\text { resultado(s) } \\
\end{array}$ \\
\hline 1 & $\begin{array}{l}\text { (Arzarello et al., 2013) } \\
\text { (Arzarello et al., 2014) } \\
\text { (Bairral et al., 2016) } \\
\text { (Bairral, 2013) } \\
\text { (Bairral et al., 2015) } \\
\text { (Bairral et al., 2017) }\end{array}$ & $\begin{array}{l}\text { Captura y análisis, } \\
\text { formas de toque y de } \\
\text { manipulación en la } \\
\text { pantalla }\end{array}$ & $\begin{array}{l}\text {-Estudiantes de } \\
\text { Educación } \\
\text { Media }^{4} \\
\text {-Futuros } \\
\text { profesores de } \\
\text { Matemática }^{5}\end{array}$ & $\begin{array}{l}\text {-Geometric } \\
\text { Constructer } \\
(\mathrm{GC})^{6} \\
\text {-Sketchometry }\end{array}$ & $\begin{array}{l}\text {-Tipos de toques } \\
\text {-Importancia del toque } \\
\text { de aproximación } \\
\text {-Existencia de dos } \\
\text { dominios (constructivo } \\
\text { y relacional) }\end{array}$ \\
\hline 2 & $\begin{array}{l}\text { (Silva, 2017) } \\
\text { (Silva \& Bairral, 2019) }\end{array}$ & $\begin{array}{l}\text { Análisis de procesos } \\
\text { de elaboración de } \\
\text { demostraciones } \\
\text { sobre cuadriláteros }\end{array}$ & $\begin{array}{l}\text {-Futuros } \\
\text { profesores de } \\
\text { Matemática }\end{array}$ & $\begin{array}{l}\text { FreeGeo en } \\
\text { Tablet }\end{array}$ & $\begin{array}{l}\text {-Dominio relacional } \\
\text { como momento } \\
\text { propicio de refinamiento } \\
\text { de conceptos, } \\
\text { propiedades, conjeturas } \\
\text { y procesos de } \\
\text { construcción de } \\
\text { demostraciones }\end{array}$ \\
\hline 3 & $\begin{array}{l}\text { (Bairral \& Silva, 2018) } \\
\text { (Bairral, 2019) }\end{array}$ & $\begin{array}{l}\text { Análisis de ideas y } \\
\text { justificaciones, } \\
\text { surgidas en la } \\
\text { identificación y } \\
\text { conceptualización de } \\
\text { cuadriláteros o de } \\
\text { algunas de sus } \\
\text { propiedades }\end{array}$ & $\begin{array}{l}\text {-Estudiantes de } \\
\text { Educación } \\
\text { Media }\end{array}$ & $\begin{array}{l}\text { FreeGeo en } \\
\text { Tablet }\end{array}$ & $\begin{array}{l}\text {-Enriquecimiento de las } \\
\text { formas de justificar y del } \\
\text { surgimiento de } \\
\text { organizaciones lógicas }\end{array}$ \\
\hline
\end{tabular}

En general, el enfoque de los estudios estuvo en el aprendizaje relacionado con la identificación y la conceptualización de los principales cuadriláteros (cuadrado, rectángulo, rombo, trapecio y un

\footnotetext{
${ }^{1}$ Agradezco a Barbara Caroline C. Chagas da Silva y a Elen Roza da Conceição Silva, implicadas directamente en los estudios cuyos extractos son presentados en este artículo.

${ }^{2}$ www.gepeticem.ufrrj.br.

${ }^{3}$ Una conferencia disponible en https://www.youtube.com/watch?v=Ptm4P4fiNaw podría enriquecer la comprensión de este artículo.

${ }^{4}$ Nivel educativo correspondiente al "Ensino Médio" de Brasil.

${ }^{5}$ En Brasil nos referimos a "Licenciatura en Matemática”.

${ }^{6}$ Recuperado de http://www.auemath.aichi-edu.ac.jp/teacher/iijima/gc/test-gcwin/download.htm.
} 
paralelogramo en general), y con el surgimiento de sus principales propiedades y de formas de justificar sus ideas y de razonar con AGDcT.

En todos los estudios fueron utilizados los smartphones o tabletas ${ }^{7}$ de los propios participantes. Cuando era necesario, proporcionamos equipos disponibles en nuestro grupo de investigación. Siempre priorizamos aplicaciones gratuitas. Al contrario del estudio 1, que fue constituido por algunas tareas, en los estudios 2 y 3 fueron construidas secuencias didácticas adecuadas a su propósito y considerando la complejidad didáctico-conceptual en función de los participantes ${ }^{8}$. En el primer estudio, invitamos aleatoriamente a estudiantes que tenían experiencias previas con AGD. En los dos últimos, el trabajo fue realizado directamente en la sala de clases -en el segundo, algunos conocían un poco un AGD, y en el tercero ninguno tenía conocimiento.

A continuación, presento algunos presupuestos teóricos que sustentan las investigaciones enfocadas en los AGDcT desarrolladas en el grupo de investigación que coordino?.

- Las tecnologías digitales móviles recrean y modifican, por medio de las interacciones, el modo cómo compartimos informaciones, nos relacionamos con el mundo y con el otro. Por lo tanto, éstas modifican nuestra subjetividad y los procesos cognitivos involucrados en una actividad matemática determinada.

- Los toques en pantalla deben ser vistos como trayectos de interacción y reveladores de aspectos del pensamiento matemático. Aunque constituyan movimientos específicos, los toques no son sinónimos de arrastres (en AGD). Éstos deben ser vistos como otra forma de manifestación de la cognición y, por lo tanto, también deben integrar el paquete semiótico y la multimodalidad comunicativa en clases de matemática. Valorar toques significa potenciar nuestra cognición corporificada y, consecuentemente, asumir los smartphones y tabletas como extensión de nuestro cuerpo (físico).

- Los procesos de demostración transitan entre dominios constructivos y relacionales. Por lo tanto, las investigaciones sobre procesos de demostración en matemática no deben orientarse únicamente hacia la validación de determinado resultado. El surgimiento y la producción de justificaciones o demostraciones deben potenciar el desarrollo de diferentes estrategias argumentativas y de aprendizaje.

- El rendimiento del dispositivo y el diseño de las tareas están entrelazados. Por lo tanto, la tarea debe ser reconfigurada integral o parcialmente en el caso de que exista un nuevo ícono o una posibilidad diferente de toque.

\footnotetext{
${ }^{7}$ Al utilizar una aplicación en el smartphone de los alumnos, tenga en mente que ellos podrán desinstalarla y en la próxima clase será necesario reinstalarla, además de correr el riesgo de perder lo que fue hecho y guardado. Busque formas de guardar continuamente los datos.

${ }^{8}$ Ver Anexo IV al final de este documento.

${ }^{9}$ Usaré el singular cuando haga referencia a posicionamientos míos y el plural cuando sean ideas colectivas y ya socializadas en publicaciones anteriores.
} 
A diferencia del orden habitual de un artículo científico (introducción, teoría, metodología, resultados y conclusiones), aquí decidí combinar aspectos teóricos y ejemplos de los tres estudios, con el propósito de facilitar la comprensión de los resultados. No todos los diálogos teóricos son oriundos de estudios con AGDcT. Algunos provienen de AGD sin toque (en adelante, AGD).

\section{TRABAJANDO CUADRILÁTEROS EN AGD}

La interacción en un ambiente de geometría dinámica (AGD) como el GeoGebra posibilita una nueva acción dinámica: la de arrastrar y desplazar objetos en una construcción. El desplazamiento dinámico no es el mismo que el acto de dibujar en papel (Bairral \& Barreira, 2017). La construcción es diferente porque ésta puede ser transformada y modificada, conservando características invariantes (Sinclair \& Yurita, 2008).

La acción de arrastrar y las construcciones proporcionan otra contribución: la diferencia entre dibujo (un ejemplo) y figura (una clase). Por lo tanto, lo que hacemos cuando construimos un cuadrado con regla y compás es un dibujo, pues éste representa un caso particular. Por el contrario, una figura representa una infinidad de objetos (Laborde, 2001). El dibujo es una representación estática, mientras que la figura involucra al usuario por medio de herramientas que permiten manipular una variedad de objetos geométricos que conservan sus propiedades (Healy \& Hoyles, 2001). Estas autoras señalan que, por el hecho de representar una clase, la figura es una representación interactiva del campo teórico, ya que ésta no sugiere únicamente un caso particular. En ese proceso, los alumnos explican sus estrategias e ideas para las construcciones geométricas realizadas en AGD, pues generalmente ellos buscan desarrollar una proposición e incluso una demostración matemática.

Aunque esa diferencia entre dibujo y figura sea un aspecto fundacional de la geometría, en AGD no hay necesidad de realizarla. La comprensión de esa singularidad cognitiva en el movimiento entre dibujo y construcción geométrica es relevante; no obstante, didácticamente, el docente puede dejar que el trabajo fluya más naturalmente, sin reforzar la ambigüedad entre figura y dibujo (Mariotti, 2019). Ciertamente, con actividades debidamente planificadas se hace necesario que se tome conciencia de las propiedades de la figura, que éstas sean explicitadas, para que sea posible realizar una imagen en la pantalla a partir de la figura. La construcción de determinados elementos implica la manutención de todo el complejo de propiedades que definen la figura geométrica representada (Mariotti, 2019).

Dalcín y Molfino (2012) propusieron actividades orientadas hacia la construcción y justificación de propiedades de cuadriláteros a partir de la clasificación de estos polígonos, realizada por los propios alumnos. Usando computadora, lápiz y papel, surgieron diversas respuestas para un mismo tipo de polígono. A pesar de la dificultad para discernir si una definición es equivalente o no a otra, los investigadores destacaron la originalidad en las clasificaciones y justificaciones realizadas por los estudiantes -algunas, incluso, diferentes de las presentadas en los libros didácticos. Los autores señalan 
que la construcción dinámica realizada en el GeoGebra facilitó la observación de las propiedades y la búsqueda de argumentos deductivos.

La clasificación de cuadriláteros constituye un campo fértil que relaciona conceptos, definiciones y propiedades geométricas. En esa relación, varias clasificaciones son posibles (Villiers, 1994). En la línea de Villiers, los estudios de Bairral y Silva (2018) y de Silva y Bairral (2019) fueron planificados con el propósito de involucrar a los participantes en procesos de definición, clasificación y comparación crítica de alternativas.

En el transcurso de las implementaciones, íbamos preguntando sobre cuadriláteros que se iban encajando en más de una clase (cuadrados, rectángulos, rombos, paralelogramos y trapecios), a medida que iban surgiendo las características de cada uno durante las actividades. Eran preguntas provocativas, con el propósito de estimular a los grupos de alumnos, pero la clasificación no era un fin en sí misma. Por ejemplo, al manipular el trapecio para obtener el trapecio isósceles en el encuentro 4, la mayoría de los estudiantes observó figuras que podrían encajarse en la clase de los rectángulos, también al ajustar la medida de los ángulos en $90^{\circ}$ exactos. Algunos aprendices llegaron a decir que ellos, con un poco más de coordinación motora para desplazar los vértices, conseguirían modificar los ángulos y obtener un rectángulo, sin la necesidad de deshacer la construcción del trapecio. En la Tabla 2 están organizadas las respuestas de dos estudiantes registradas en la ficha 4 (Anexo V) sobre trapecios.

\section{Tabla 2}

Respuestas de la ficha sobre trapecios (Estudio 3) (Bairral y Silva, 2018, pp. 175-176)

\begin{tabular}{|c|c|c|}
\hline \multirow{2}{*}{ Pregunta } & \multicolumn{2}{|c|}{ Respuestas } \\
\hline & Caroline & Victor \\
\hline 2 & Dos paralelos que no se cortan. & Un par de paralelos. \\
\hline 3 & $\begin{array}{l}\text { Siempre que los lados se mueven, los números } \\
\text { cambian, según se mueven los lados. }\end{array}$ & Al mover la medida de los lados, cambia. \\
\hline 4 & $\begin{array}{l}\text { Los lados opuestos siempre quedan iguales, ya los } \\
\text { lados de los ángulos no permanecen en lados } \\
\text { iguales. }\end{array}$ & $\mathrm{Al}$ mover los ángulos, aumentan y disminuyen. \\
\hline 5 & $\begin{array}{l}\text { Las paralelas siempre permanecen en lados } \\
\text { totalmente diferentes. }\end{array}$ & $\begin{array}{l}\text { Que los ángulos y los lados no son de la misma } \\
\text { medida. }\end{array}$ \\
\hline 6 & 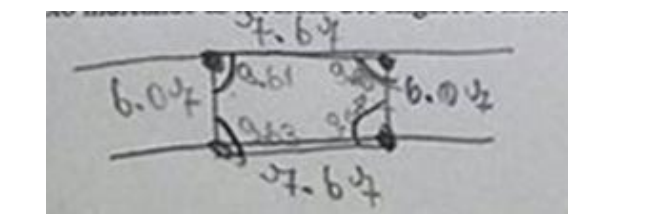 & $\begin{array}{l}96.09 \\
\frac{1925}{71.27}\end{array}$ \\
\hline 7 & $\begin{array}{l}\text { Yo observo que los ángulos son totalmente } \\
\text { diferentes. }\end{array}$ & Que son todos diferentes. \\
\hline
\end{tabular}

En la pregunta 5, ambos estudiantes destacan como características de los trapecios: los ángulos y lados no necesitan tener la misma medida; y las paralelas permanecen siempre paralelas. Otro alumno, 
Patrick, también resaltó una característica de forma bien contundente: "Por más que las mueva, las paralelas del trapecio nunca dejan de existir”.

En la pregunta 6, algo interesante que observar es la diferencia entre los dibujos obtenidos en las respuestas: durante la aplicación, los alumnos percibieron que podían modificar el trapecio a un rectángulo, como la alumna Caroline intentó mostrar. Ellos bromearon, diciendo que no tenían coordinación motora para ajustar los ángulos, con los movimientos de sus dedos, hasta que todos tuvieran una medida de $90^{\circ}$, pero aun así plantearon esa observación, incluyendo al rectángulo en los trapecios durante la conversación en la sala. También es interesante ese registro de medidas usando numeración decimal, favorecido por el dispositivo, cosa que en un dibujo hecho a mano alzada posiblemente no sería observada. En la pregunta 7, los dos alumnos dijeron que tenían todos los ángulos diferentes. Aparentemente, ellos no prestaron atención al hecho de que en la pregunta se estaba haciendo referencia a las medidas de los ángulos del trapecio rectángulo, que posee dos ángulos iguales a $90^{\circ}$. Es posible que hayan hecho referencia al trapecio obtenido anteriormente.

Es notorio que en AGDcT, el proceso de aprendizaje se desarrolla a medida que el sujeto internaliza -a partir de una forma estática o en movimiento- ciertos objetos (conceptos, formas, propiedades, etc.), con el fin de producir significados personales mediante los toques en la, con o a partir de la pantalla del dispositivo (Bairral, 2017, 2020). La conjunción en las diferentes formas de manifestación del aprendizaje y del lenguaje (habla, expresiones gestuales, toques en pantalla) o de registro (diagramas y otros registros escritos, pantallas con construcciones en AGD) da indicios de la internalización de los artefactos semióticos construidos, explorados y manipulados en la pantalla del dispositivo cuando estudiantes lidian con formas geométricas ( $\mathrm{Ng} \&$ Sinclair, 2015).

Mientras que en AGD es común arrastrar, en AGDcT podemos tener diferentes tipos de toques. Todas las acciones son importantes. Como señala Mariotti (2000, 2019), en el movimiento de arrastre, mediante clic en el ratón, el resultado es verdadero y la proposición puede ser demostrada a partir de una prueba geométrica. Además, los procedimientos lógicos utilizados también requieren ser considerados y aceptados. La autora sostiene que la relación entre una construcción y el teorema que le otorga validez es generalmente compleja, y no absolutamente inmediata. Es importante considerar también la construcción geométrica como elemento de la teoría, complementa Mariotti (2019).

\section{DE ARRASTRES EN EL RATÓN A TOQUES EN PANTALLA}

Las manipulaciones en la pantalla (con ella o a partir de ella $)^{10}$ en un dispositivo poseen intenciones y, de la misma forma que los gestos y otras manifestaciones en el ámbito de la oralidad, producen sentidos en un determinado contexto de comunicación. Las manipulaciones directas en la

\footnotetext{
${ }^{10}$ Siempre que me refiera a manipulaciones, considere una de estas posibilidades: en la pantalla, con la pantalla o a partir de la pantalla.
} 
pantalla constituyen lo que denominamos toques. Estos toques involucran seis acciones básicas con los dedos (Choi, 2008, citado en Park et al., 2011): toque ${ }^{11}$ (tap), toque doble (double tap), toque prolongado (long tap / hold), arrastre (drag), cambio de pantalla (flick) y toques múltiples (girar, rotar), como se ilustra a continuación.

\section{Figura 1}

Tipos básicos de toques (Choi, 2008, citado en Park et al., 2011, p. 841)

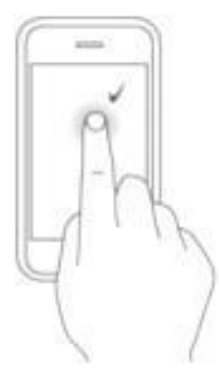

Tap

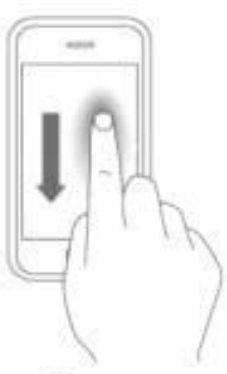

Drag

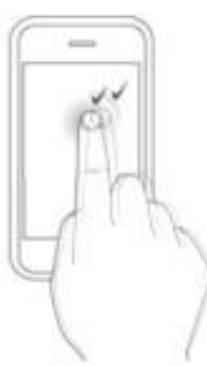

Double Tap

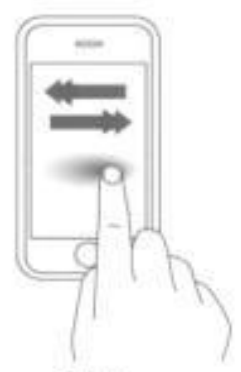

Flick

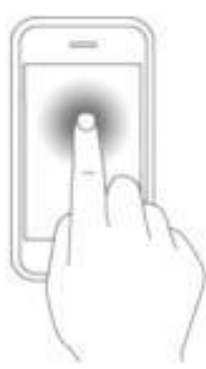

Long Tap

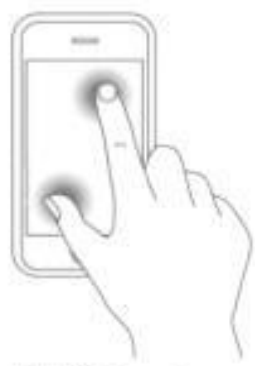

Multi-touch

Estos seis tipos de toques pueden tener variaciones de función, de acuerdo con el sistema operativo (iOS, Android, etc.) o del dispositivo. Además, dada la simultaneidad de movimientos y cambios en objetos construidos, los toques en pantalla deben ser vistos como trayectos de interacción y no como los puntos o clic en zonas de la construcción.

En el estudio 1, una de las tareas solicitaba a los alumnos de Educación Media que, al trabajar en el Geometric Constructor (GC), demostraran que la figura EFGH, cuyos vértices son los puntos medios de los lados del cuadrilátero $\mathrm{ABCD}$, era un paralelogramo. Como la actividad tenía un propósito (verificar y justificar la propiedad), la manipulación por aproximación fue muy recurrente para analizar el comportamiento de la forma construida como un todo y de sus elementos invariantes. Cuando los estudiantes necesitaban realizar construcciones (de diagonales, ángulos y paralelas), el uso de toques fue bastante observado, como se muestra en la Tabla 3.

${ }^{11}$ Golpe ligero. 


\section{Tabla 3}

Ilustración de parte del razonamiento de una pareja en la tarea (Estudio 1) (Bairral, 2013)

\begin{tabular}{ll}
\hline Tipo de toque & \multicolumn{1}{c}{ Razonamiento } \\
\hline untalla del GC & $\begin{array}{l}\text { Ajustando la forma de la figura inicial para } \\
\text { un rectángulo }\end{array}$ \\
\hline
\end{tabular}

Los seis tipos de toques mostrados en la Figura 1 llegaron a mostrarse muy apropiados al inicio de nuestra investigación y al ambiente utilizado, el Geometric Constructor. Incluso, fueron observados en otros dispositivos que usamos. Sin embargo, en términos de pensamiento geométrico, fue posible avanzar en esa categorización, proponiendo dos modos de arrastre (libre y de aproximación) y posibilidades de uso de los dedos para girar una figura o partes de ésta.

En el Anexo VII se observa la tipología ampliada (Arzarello et al., 2013) a partir de Choi (2008), y en el Anexo VIII se encuentran detalles para tareas que involucran isometrías (Assis, 2020). De todos modos, la preocupación por el tipo de toque no es lo que debe orientar nuestra dinámica de enseñanza, pero cabe resaltar aquí que los toques revelan aspectos del pensamiento matemático, y esa forma de cognición también enriquece los procesos de elaboración de justificaciones y demostraciones matemáticas, entre otros, como se indica en la Tabla 4. 
Tabla 4

Desarrollo del pensamiento geométrico de estudiantes en el GC(Estudio 1) (Bairral et al., 2016)

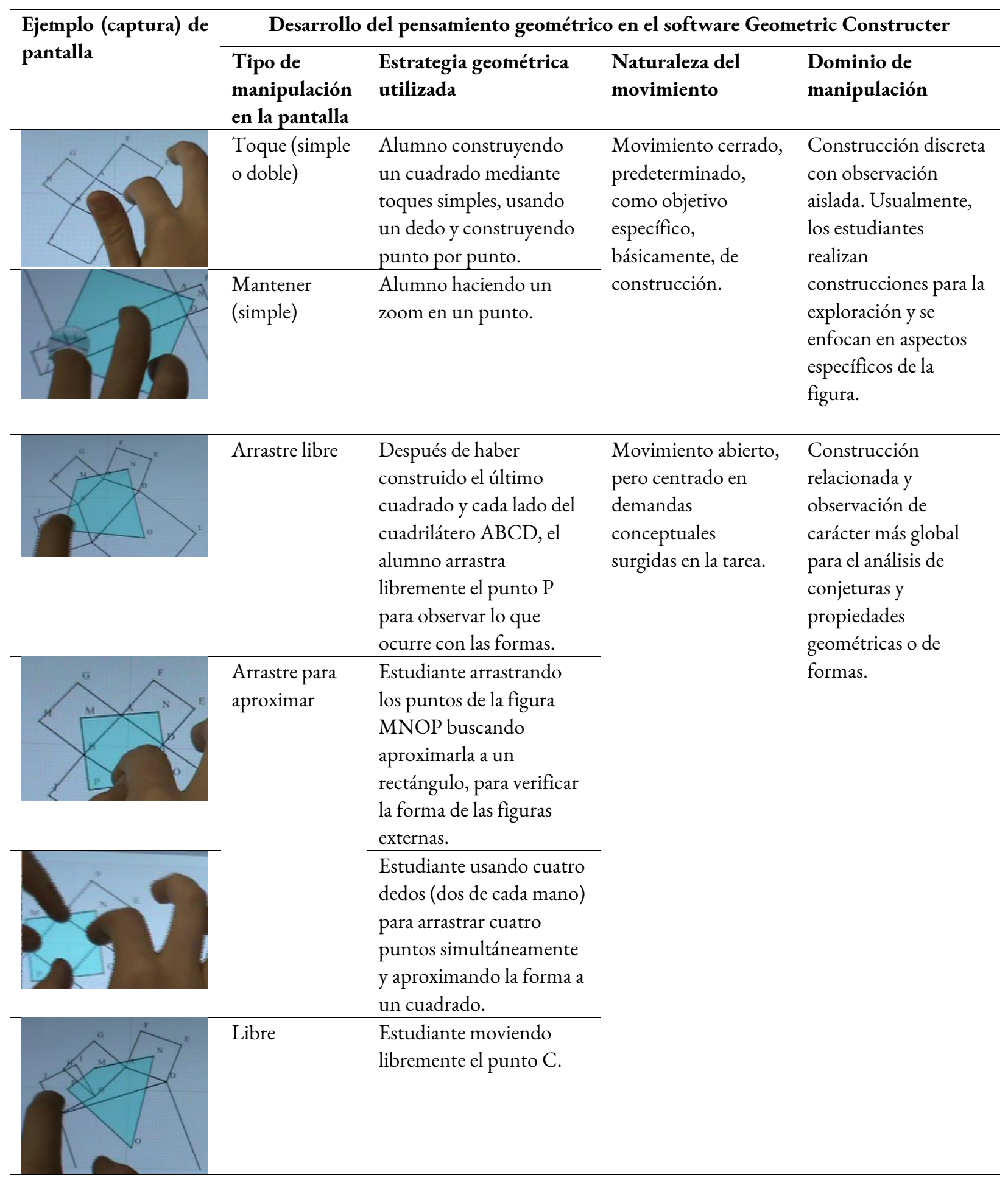

Las manipulaciones de pantalla táctil (touchscreen) constituyen un campo fértil de exploración en la investigación educativa, especialmente, porque éstas dan lugar a nuevas inspiraciones de prácticas 
formativas con dispositivos móviles. En cualquier contexto formativo, las tareas adquieren importancia en la construcción del conocimiento. Por lo tanto, su adaptación a las especificidades de cada dispositivo es primordial. Saber la importancia cognitiva del tipo de manipulación en la pantalla es importante para que el docente diseñe tareas en las que el estudiante pueda realizar las manipulaciones adecuadamente.

\section{TIPOS DE TOQUE EN PANTALLA Y DOMINIOS DE DESARROLLO DEL RAZONAMIENTO}

Estamos asumiendo el concepto de procesos de elaboración de demostraciones como las diversas justificaciones y representaciones que componen la producción de argumentos ${ }^{12}$ y pruebas matemáticas, incluyendo los recursos tecnológicos digitales, la producción del alumno, su discurso, sus gestos y demás formas de expresión del lenguaje. Defendemos que los procesos de demostración en dispositivos con toques en pantalla transitan dialécticamente entre dos dominios reflexivos importantes: constructivo y relacional.

Bairral et al. (2017) destacan dos dominios de manipulaciones de pantalla táctil en sus investigaciones: el constructivo, que involucra toques simples y específicos en la pantalla (como toques dobles, por ejemplo) y observaciones aisladas, generalmente, en momentos de construcción de objetos matemáticos o de verificación de una observación puntual; y el relacional, que comprende la combinación y articulación de múltiples toques más elaborados o no, por ejemplo: el movimiento simultáneo de aproximación y arrastre, o solamente el de aproximación. En este último dominio, la consideración de aspectos globales en la observación del diagrama dinámico es una característica marcada. Además, la conjetura es un tipo de acción del pensamiento específico del dominio relacional. La Tabla 5 ilustra ejemplos de toques predominantes en cada dominio.

\section{Tabla 5}

Toques y dominios de razonamiento (Silva, 2017, p. 16)

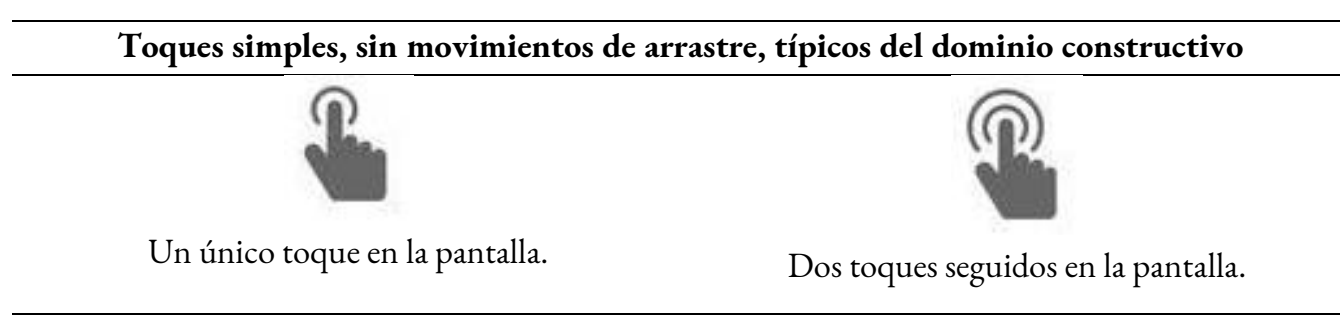

Toques elaborados, con movimientos de arrastre, típicos del dominio relacional

$\begin{array}{cc}\text { Manipulación de arrastre para arriba y para } & \text { Manipulación de arrastre para la derecha, sin } \\ \text { abajo, sin retirar el dedo de la pantalla. } & \text { retirar el dedo de la pantalla. }\end{array}$

\footnotetext{
${ }^{12}$ Argumento será entendido como cuestionamiento, desacuerdo o duda que desemboca en un proceso de negociación que ayuda a aclarar la idea emergente.
} 
Según Bairral et al. (2017), en el dominio constructivo la mirada de los sujetos se dirige hacia aspectos puntuales del objeto geométrico explorado en la aplicación, esto es, hacia momentos en que las manipulaciones realizadas en la pantalla poseen la función de medir, construir, editar y nombrar elementos. Como el proceso de creación de un objeto geométrico en AGDcT involucra la selección de herramientas específicas de la aplicación, las manipulaciones de toques son más frecuentes. Sin embargo, estos investigadores señalan que el razonamiento en este dominio está orientado hacia una mirada específica de un elemento del objeto explorado.

En el dominio relacional, las manipulaciones en la pantalla pasan a tener la función de deformar (o no) la figura por medio del arrastre, aproximar el objeto a figuras familiares y aumentar o disminuir la figura. Específicamente sobre el razonamiento, los autores destacan el surgimiento de conjeturas, hipótesis, observación de relaciones, cuestionamientos, refinamientos, etc., como elementos pertinentes al dominio relacional.

Silva (2017) usó el Sketchometry, el FreeGeo y el GeoGebra en smartphones y tabletas con futuros profesores de Matemática y destacó la construcción dinámica en pantallas, la movilidad, el compartir en red y la práctica dialógica como características de clases que potencian el proceso argumentativo y el procedimiento de organización de una demostración. El análisis también evidenció que el movimiento entre los dominios ${ }^{13}$ de manipulación (constructiva y relacional) en pantalla se mostró favorable al proceso de refinamiento del razonamiento y de elaboración de una demostración sobre cuadriláteros. Silva destacó aspectos importantes en el proceso de elaboración de demostraciones, tales como la observación, exploración, verificación, formulación de hipótesis y refinamiento conceptual o de propiedades.

En AGDcT, la exploración y la conjetura son importantes en los procesos de demostración. Por ejemplo, en el dominio relacional se percibe que el refinamiento de conjeturas se hace más presente, ya que los movimientos de arrastre realizados en la pantalla buscan generalmente la aproximación del objeto a algo conocido por el estudiante. En esos momentos, la mirada del futuro profesor hacia la variación dinámica del diagrama en la pantalla observa relaciones y propiedades. En el ámbito constructivo, tienden a predominar aspectos puntuales de construcción y de verificación en el razonamiento de los sujetos.

Los dos dominios son importantes y están interrelacionados. Lo que es construido pasa a ser explorado, analizado, refinado o refutado. Ambos involucran pensamiento. Generalmente, es en el dominio relacional que surgen nuevos hallazgos matemáticos. Aunque en el contexto constructivo contamos con toques (simples, dobles, etc.) en la pantalla, puede que éstos no sean necesarios en el

${ }^{13}$ Véase más detalles sobre esos dominios y procesos de demostración en Bairral et al. (2016). 
dominio relacional. No obstante, en este último dominio son recurrentes arrastres y movimientos para aproximar figuras.

En un contexto de trabajo en grupos, los dominios de manipulación (con o a partir de la pantalla) ejercen influencia en el razonamiento de todos los involucrados, y no solamente de aquel que manipula la aplicación. La interacción asume también un papel de observación compartida, pues nuestro cerebro realiza mapeos que permiten al observador hacer inferencias o anticipaciones de hallazgos, ya que, en ese acto de observar, éste también está pensando. La dinámica dialógica de manipulaciones diversas y de observación compartida entre los implicados es potenciada por la manipulación (con o sin toques en la pantalla) de un AGDcT.

\section{DESARROLLO DE DEMOSTRACIONES MATEMÁTICAS Y DOMINIOS DE RAZONAMIENTO}

El uso de AGDc'T en demostraciones y procesos de justificaciones o pruebas matemáticas puede constituir un camino potente y diferenciado en el aprendizaje de los alumnos. La producción de demostraciones en la formación inicial en matemática constituye, en la mayoría de los casos, una dificultad para los futuros profesores. Ese obstáculo, en la concepción de Cirillo y Herbst (2012), puede justificarse por los aspectos históricos y culturales que permean la enseñanza de la matemática. Estos autores afirman que, en general, tendemos a enseñar del mismo modo como nos enseñaron, un círculo vicioso de una práctica pautada en el rigor estático de verdades absolutas, que no se exploran ni se investigan, que no se cuestionan o relativizan, que no producen demostraciones diferentes de los formatos clásicos presentados en los libros didácticos. Estos investigadores llaman la atención sobre la ampliación del papel del alumno como un sujeto más activo en las demostraciones geométricas.

Para Villiers (2001, p. 31), "tradicionalmente la función de la demostración ha sido vista exclusivamente como una referencia para la verificación de la exactitud de las afirmaciones matemáticas”. Esa visión sólo servía para despejar dudas personales o de escépticos. El papel docente es importante para desafiar al alumno a buscar, conjeturar, argumentar y explorar las diferentes funciones que la demostración matemática nos puede proporcionar. En línea con el autor, defendemos que la demostración matemática puede asumir, también en AGDcT, funciones auténticas de verificación, explicación, sistematización, hallazgos, comunicación y de desafío intelectual.

Lasa y Wilhelmi (2013), al trabajar con profesores de Educación Básica en España, afirman que la incorporación de un AGD en la enseñanza se debe realizar gradualmente, siguiendo tres momentos: exploración, ilustración y demostración de una propiedad geométrica. En el diseño de nuestras implementaciones, siempre adoptamos el momento de la familiarización. En ese momento, es importante dejar que los sujetos manipulen libremente el dispositivo. Éstos pueden realizar construcciones aleatorias (rectas, segmentos, etc.) para familiarizarse con tareas como la del Anexo II. 
En la familiarización, el individuo se integra al escenario y no sólo se involucra en la realización de una actividad específica. La familiarización no es una comprobación, sino un momento de inmersión en el escenario, a pesar de que pueda haber una actividad más exploratoria, por ejemplo, construir un círculo, después manipular y hacer observaciones. Lo que se produce en la familiarización es también objeto de estudio y de aprendizaje, o sea, no es una producción aislada. La familiarización no es un momento de dominio integral de herramientas del AGD. Incluso ese dominio, cuando ocurre, demora un tiempo. Inicialmente, el docente puede ir dando prioridad a algunas herramientas del ambiente para las tareas que implementa y puede dejar que el alumno vaya descubriendo y utilizando otras a lo largo del proceso.

Dalcín y Molfino (2012), en su investigación en la formación inicial de profesores de Matemática y con el GeoGebra en Uruguay, proponen actividades que posibilitan la construcción y justificación de propiedades de cuadriláteros que surgen a partir de la clasificación de estos polígonos, realizada por los propios alumnos. Al solicitar que los estudiantes definieran algunos cuadriláteros, surgieron diversas respuestas que dieron lugar a diferentes clases para un mismo tipo de polígono. Además de la dificultad que los discentes presentan para discernir si una definición es equivalente o no a otra, así como para dibujar tales cuadriláteros, el modelo de figuras con lados paralelos a los márgenes de la hoja fueron los más frecuentes. Sus resultados destacan la originalidad en las clasificaciones y justificaciones que surgieron de los propios estudiantes en el transcurso de las implementaciones: éstos proponen clasificaciones y definiciones diferentes, incluso, de las presentadas en los libros de texto. La construcción dinámica realizada en el GeoGebra facilitó la observación de las propiedades y la búsqueda de argumentos deductivos.

De acuerdo con Mariotti (2000), los alumnos presentan dificultad en la sistematización teórica del conocimiento inductivo, ya que éstos cuestionan porqué propiedades bien conocidas deben ser demostradas. Los argumentos son usados para apoyar su verdad, que es evidente para los estudiantes. Esta dificultad está vinculada a la enseñanza de la geometría mediante un conjunto de definiciones que describen figuras geométricas y proposiciones, indicando propiedades que, muchas veces, tienen un alto grado de evidencia. Según la investigadora, el abordaje deductivo involucra dos aspectos entrelazados: por un lado, la necesidad de justificación; y, por otro, la idea de un sistema teórico dentro del cual esa justificación puede convertirse en una demostración. La autora también destaca que la relación con el conocimiento geométrico es modificada a través de la mediación ofrecida por las peculiaridades del AGD. Esta transformación está relacionada estrictamente con el paso de una geometría intuitiva -una colección de propiedades evidentes- a una geometría deductiva, un sistema de relaciones entre afirmaciones, validado por la demostración. 


\section{TOQUES Y REGISTROS EN LA IDENTIFICACIÓN DE CUADRILÁTEROS}

A continuación, ilustro algunos ejemplos de respuestas de alumnos de Educación Media a la tarea de construir un rombo en el FreeGeo y de realizar observaciones sobre sus lados, ángulos y diagonales (estudio 3). Son respuestas en actividades introductorias al tema, que no había sido estudiado por los alumnos, quienes tampoco habían tenido experiencia previa con la aplicación.

Todos sus lados son iguales.

Angulos diferentes.

Diagonales iguales.

Sus lados están formados por dos pares de rectas paralelas.

Sus diagonales tienen sus ángulos iguales.

Las respuestas anteriores son aquellas comúnmente observadas en los libros didácticos, cuando caracterizamos ese tipo de cuadrilátero. Eso ya era esperado, dado que las actividades introductorias de la secuencia de actividades (Anexo I) contenían, en sus alternativas, pistas sobre propiedades y conceptos geométricos, comúnmente presentes en libros didácticos. Por lo tanto, ésa es una visibilidad de la influencia de la medición semiótica, que no debe ser vista como un problema. El desafío para el profesor es no crear expectativas de respuestas, ya que su propósito debe ir más allá de ello.

Sinclair y Robutti (2013) discuten la naturaleza epistemológica y cognitiva de algunas herramientas de los AGD y sus relaciones con los procesos de demostración. Ellas sostienen que necesitamos nuevos modelos o rutas de aprendizaje que puedan dar cuenta del impacto de los AGD en el desarrollo del pensamiento del alumno. Una vez más, el papel del docente se destaca como un elemento crucial en este contexto, ya que él es responsable por enfatizar las relaciones entre los objetos, durante el intento de introducir a los alumnos en el mundo teórico de la geometría. Para las autoras, es necesario profundizar las investigaciones de cómo el profesor puede subsidiar y lidiar con las implicaciones cognitivas y epistemológicas de las herramientas en este tipo de ambiente.

$\mathrm{Ng}$ y Sinclair (2015) reportaron dos actividades con las que se buscaba abordar la idea de cizallar polígonos en AGDcT con alumnos de Educación Media. Sus resultados indican que las producciones de gestos, diagramas en papel y el propio discurso de los alumnos dan indicios de la internalización de los artefactos semióticos construidos, explorados y manipulados en la pantalla del dispositivo. La forma de dar cuenta de esa diversidad es colocar la atención en la mediación semiótica (Bussi \& Mariotti, 2008).

Ese concepto vigotskiano permite una mirada cognitiva sobre la función de un instrumento, en este caso, los AGDcT, en la construcción del conocimiento matemático (Mariotti, 2019). Didácticamente, la atención en la mediación semiótica es importante en la construcción de significados que el profesor imagina surgir en sus objetivos, ya que el pasaje del uso del instrumento a la construcción de un significado matemático también tiene relación con la intervención docente, que ocurre en las 
interacciones con los alumnos, en las tareas propuestas y en su implementación. En otras palabras, lo que el docente planifica y ejecuta ejerce influencia en lo que el alumno puede aprender.

En un AGDcT, el diseño de una tarea tiene relación directa con el rendimiento (Assis \& Bairral, 2019) del dispositivo. Además, el objetivo de la tarea, como instrumento de mediación semiótica, tendrá implicaciones diferentes en el aprendizaje. Por ejemplo:

- Usando lápiz y papel, podemos plantear la tarea: $\underline{\operatorname{Identifica}}^{14}$ rombos en las siguientes figuras:

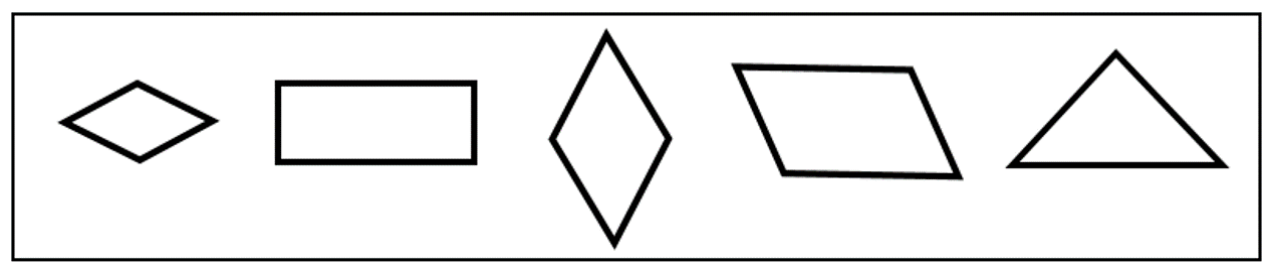

- Usando el FreeGeo, tendríamos: Construye en el FreeGeo un rombo y realiza tres observaciones: sobre sus lados, ángulos y diagonales.

Aunque sea importante y realizable con lápiz y papel, véase que observar sería una acción reducida de potencial matemático, si utilizáramos el software. En este recurso, acciones como construir; mover, observar y analizar; comparar son más providenciales y prometedoras en términos de aprendizaje. Ésas son acciones más propicias en contextos de demostración.

Las respuestas presentadas por los estudiantes (Tablas 6 y 7 ) son ilustrativas de que cambiar el recurso didáctico implica alterar la propuesta de la actividad y, consecuentemente, tendremos contenidos y aprendizajes diferentes. La respuesta a continuación se enfoca sobre la acción de mover (arrastrar). En ésta es visible el aspecto dinámico, de movimiento, de la aplicación y el hallazgo del estudiante, quien ofreció esta observación: "Moviendo $\operatorname{los}^{15}$ lados del rombo éste se transforma en otras figuras como triángulo y cuadrado”. Las siguientes respuestas también son interesantes, pues no son frecuentes en las observaciones iniciales de los alumnos:

Los ángulos de las laterales del rombo son diferentes de los de arriba y abajo y, con respecto a las diagonales, tienen la misma medida de ángulo arriba y abajo de la diagonal.

Un rombo tiene un par de ángulos obtusos y un par de ángulos agudos.

Las respuestas anteriores y la Tabla 6 son ejemplos de lo que Bairral y Barreira (2017) nos invitan a pensar: en $\mathrm{AGD}$, los puntos, las rectas y los planos dejan de ser sólo entes primitivos. Éstos no existen

\footnotetext{
${ }^{14}$ Cuando las tareas sean colectivas, recomendamos el uso de los verbos en plural. Esto no es un mero detalle ortográfico, sino una estrategia del diseño de la tarea y, consecuentemente, de la mediación semiótica.

${ }^{15}$ Subrayado nuestro.
} 
por sí solos, sino que coexisten con una variedad de objetos y construcciones geométricas, todos representados y desplazables en la pantalla. Por lo tanto, según estos autores, la geometría producida en un AGD como el GeoGebra es otra, dado que contamos con nuevas formas de manifestación del lenguaje (íconos variados, formas de medición, observación simultánea de propiedades y formas, etc.), de hallazgos y verificación de propiedades -la geometría de los movimientos en AGD, con o sin toques en pantalla.

Entendemos que la transición del uso del instrumento a la construcción de un significado matemático es dinámica y retroalimentada. Por lo tanto, los toques en pantalla deben integrar el paquete semiótico (Arzarello et al., 2009) y dinámico de objetos y relaciones entre ellos (Bairral \& Powell, 2015). Éstos también propician el surgimiento de varias formas de construcción, exploración, convencimiento y razonamiento, individual o colectivamente.

La Tabla 6 sintetiza respuestas pautadas en propiedades aparentemente guiadas por las indicadas en las actividades y ejemplos asociados a la manipulación del dispositivo, particularmente, por su posibilidad de movimiento.

\section{Tabla 6}

Sintesis de respuestas de los alumnos (elaborado a partir de Bairral, 2019)

\begin{tabular}{lll}
\hline Encuentro & $\begin{array}{c}\text { Ejemplos de respuesta pautada en propiedades } \\
\text { explicitadas en las fichas de actividades }\end{array}$ & \multicolumn{1}{c}{$\begin{array}{c}\text { Ejemplos de respuesta guiada por la } \\
\text { manipulación del software }\end{array}$} \\
\hline 2 & "Todos sus lados son iguales." & $\begin{array}{l}\text { "Moviendo los }{ }^{16} \text { lados del rombo éste se } \\
\text { transforma en otras figuras como triángulo y } \\
\text { cuadrado." }\end{array}$ \\
\hline 3 & "Que los ángulos opuestos siempre tienen el mismo & "Observé que las medidas cambian cuando se \\
& valor.” & $\begin{array}{l}\text { mueve y también los valores son iguales en los } \\
\text { lados opuestos.” }\end{array}$ \\
\hline 4 & "Las paralelas siempre permanecen en lados & $\begin{array}{l}\text { "Por más que las mueva, las paralelas del trapecio } \\
\text { nunca dejan de existir.” }\end{array}$ \\
\hline
\end{tabular}

Aunque los extractos ejemplifiquen registros escritos, éstos no pueden ser interpretados aisladamente, ya que hay imágenes que están siendo evocadas, procesadas, guiadas por construcciones y movimientos en el AGDcT. Estas formas de discurso expresan movimientos y rutinas en constante interacción con el dispositivo y que transitan entre particularidades y generalizaciones en sus hallazgos matemáticos (Ng, 2016). Por lo tanto, los toques en pantalla deben integrar ese sistema y no pueden ser entendidos como sinónimos de gestos en arrastres, tal como es considerado por $\mathrm{Ng}$ (2016).

Las dos columnas de la Tabla 6 tampoco pueden ser analizadas por separado ya que, en el proceso de mediación semiótica, el hecho de que un instrumento corporifique un saber lo torna utilizable según una doble función: por un lado, el uso del instrumento permite realizar una tarea determinada; por el

\footnotetext{
${ }^{16}$ Subrayados de los investigadores, para mostrar acciones y reflexiones intrínsecas a la manipulación del software.
} 
otro, ofrece, a quien lo usa, una vía de acceso a aquel saber que lo corporifica (Mariotti, 2019). Los subrayados son marcadores que ilustran cómo estos procesos se entrelazan y van enriqueciendo el repertorio matemático de los involucrados.

\section{TOQUES Y REGISTROS EN LA MEJORA DE LOS PROCESOS DE DEMOSTRACIÓN EN LA LICENCIATURA}

La actividad a continuación constituye un extracto de una secuencia didáctica llevada a cabo con futuros profesores de matemática (Estudio 3). La Tabla 7 detalla esta actividad.

\section{Tabla 7}

Actividad en el AGDcT (Silva, 2017, p. 73)

\section{Tarea}

2 - Abre el archivo en la App FreeGeo

a) ¿Qué tipo de cuadrilátero fue construido en la aplicación?

b) ¿Cuál es la relación existente entre las diagonales del cuadrilátero presentado en la aplicación?

c) Elabora una proposición para esa relación y demuéstrala.

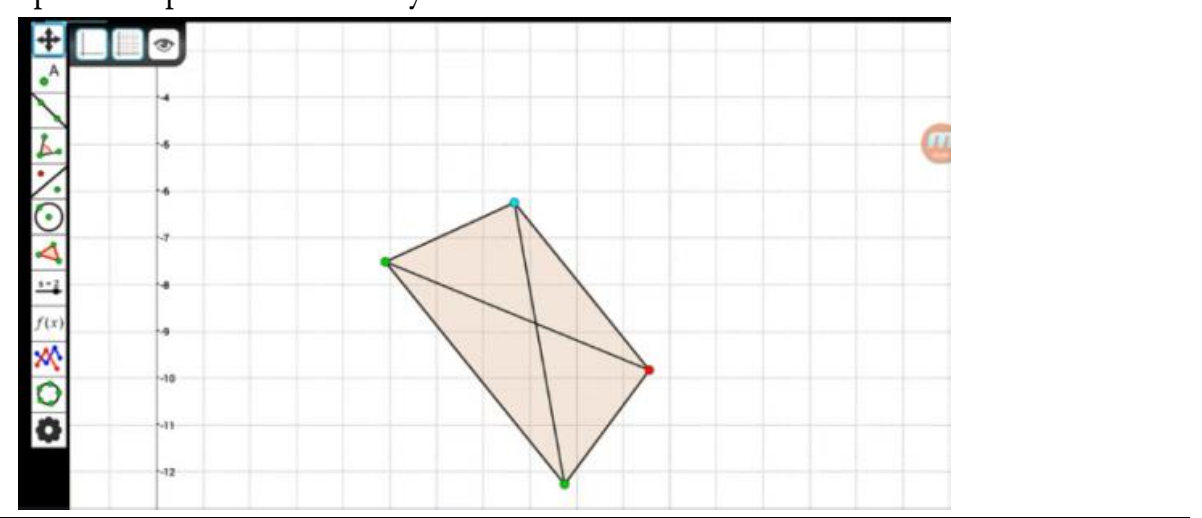

Para el diseño de esta actividad, consideramos la idea de Cirillo y Herbst (2012) en el sentido de proponer a los futuros profesores caminos inversos para llegar a la elaboración de una demostración matemática. O sea, tradicionalmente, presentaríamos la proposición y solicitaríamos que los alumnos la demostraran. Inspirados en estos autores, iniciamos la actividad por medio de la exploración de un diagrama en el AGDcT, previamente elaborado por la investigadora que condujo este estudio.

Además de ello, el tema de la tarea surgió de las dificultades de los futuros profesores. Como observamos que las definiciones ${ }^{17}$ y las propiedades del trapecio parecían confusas en actividades

\footnotetext{
${ }^{17}$ Un trapecio puede ser definido como un cuadrilátero con solo un par de lados paralelos o con, por lo menos, un par de lados paralelos. Estas dos definiciones no son muy conocidas por los futuros profesores y es importante que éstas sean bien discutidas en la Licenciatura en Matemática. Posterior a esa discusión, se puede asumir a una de esas definiciones o dejar que
} 
anteriores, invertimos un poco más en este asunto. Veamos en la Tabla 8 un fragmento de vídeo sobre esa actividad. En la tabla, los índices A1, A2 y A3 son referencias a los discursos de los alumnos, mientras que $\mathrm{P}$ se refiere al discurso de la investigadora, y las flechas en rojo indican la ubicación del toque del sujeto en la pantalla.

\section{Tabla 8}

Diálogo del grupo 2 sobre la actividad (Parte 1) (Silva, 2017, p. 74)

\section{Transcripción: 00:00:08 - 00:00:57}

A1: Nosotros estamos moviendo aqui y percibimos que se puede transformar en otro tipo de cuadrilatero. (habla simultánea a la manipulación) Mire, puede ser un rectángulo... ¿eso es un rectángulo? (tono muy bajo)

P: ¿Pero qué tipo de cuadrilátero es ése?

A1: Quétipo...

P: Si, tú dijiste asi: "se puede transformar en otro tipo".

A2: Es transformar en otro formato.

A1: :Si!' Mire, aqui es un rectángulo.

A3: No. Es un trapecio.

A1: ¿Un trapecio todavia?

A3: Todavia es un trapecio.

A1: ¿Siempre va a ser un trapecio?

A3: $S i$.

A1: Parece un rectángulo.

A3: No lo es. Es un trapecio.

A1: ¿Por qué?

A2: Porque ésas de aqui... no son fijas (tono muy bajo). Por causa del ángulo, que no es de noventa grados, ¿cierto? (parece señalar algo en el diagrama y cuestiona a $\mathrm{A} 3$, pero no tenemos la grabación del grupo, solamente de la pantalla de la tablet y el audio).

A3: Si. Ellas no son paralelas.

A1: Entiendo. Entonces, isiempre será un trapecio!
Imágenes de la pantalla
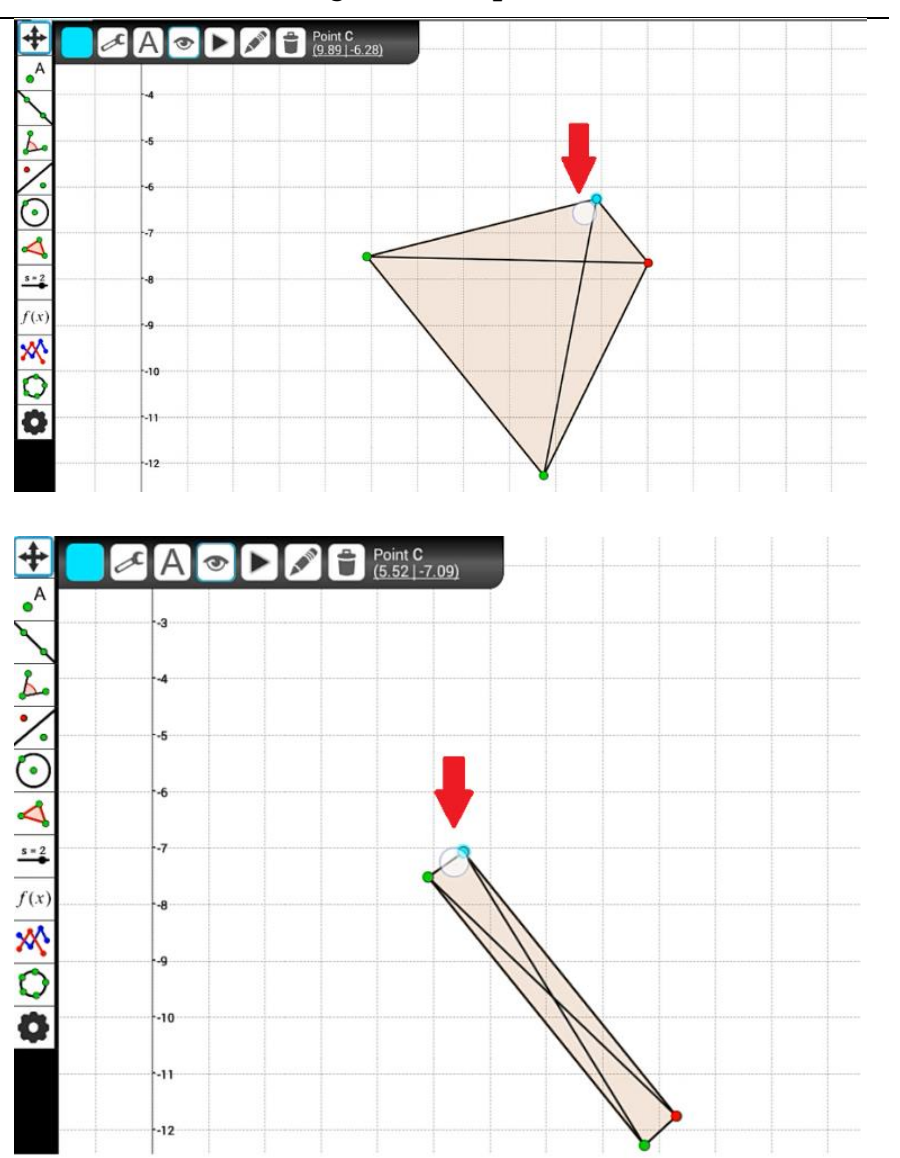

En el fragmento de vídeo (Tabla 8), observamos que las inferencias que pueden ocurrir en el dominio relacional de manipulación no necesariamente son realizadas por el alumno que manipula la pantalla, es decir, en un contexto de trabajo en grupo, los integrantes que no poseen el control momentáneo también observan la pantalla y plantean sus conclusiones con base en la observación de la manipulación del otro. Esto lo podemos observar en las siguientes intervenciones: "A1: iSi'! Mire, aqui es un rectángulo", "A3: No. Es un trapecio”, "A2: Porque ésas de aqui... no son fijas (tono muy bajo).

el trabajo fluya a partir de las clasificaciones que los sujetos fueren construyendo. Para más detalles sobre clasificaciones de cuadriláteros, véase Villiers (1994). 
Incluso, en la intervención "Por causa del ángulo, que no es de noventa grados, ¿cierto?", suponemos que quien estaría manipulando la pantalla era el alumno 1 (A1) (porque fue ese alumno quien inició la conversación con la investigadora); no obstante, los otros dos integrantes del grupo (A2 y A3) tenían la atención colocada en los cambios del objeto mientras éste era arrastrado por A1, tanto así que llegaron a alertar y explicar a éste último que su suposición de que el cuadrilátero fuese rectángulo estaba equivocada. En la Tabla 9 presentamos un fragmento más de vídeo referido a esa actividad.

\section{Tabla 9}

Diálogo del grupo 2 sobre la actividad (Parte 2) (Silva, 2017, p. 75)

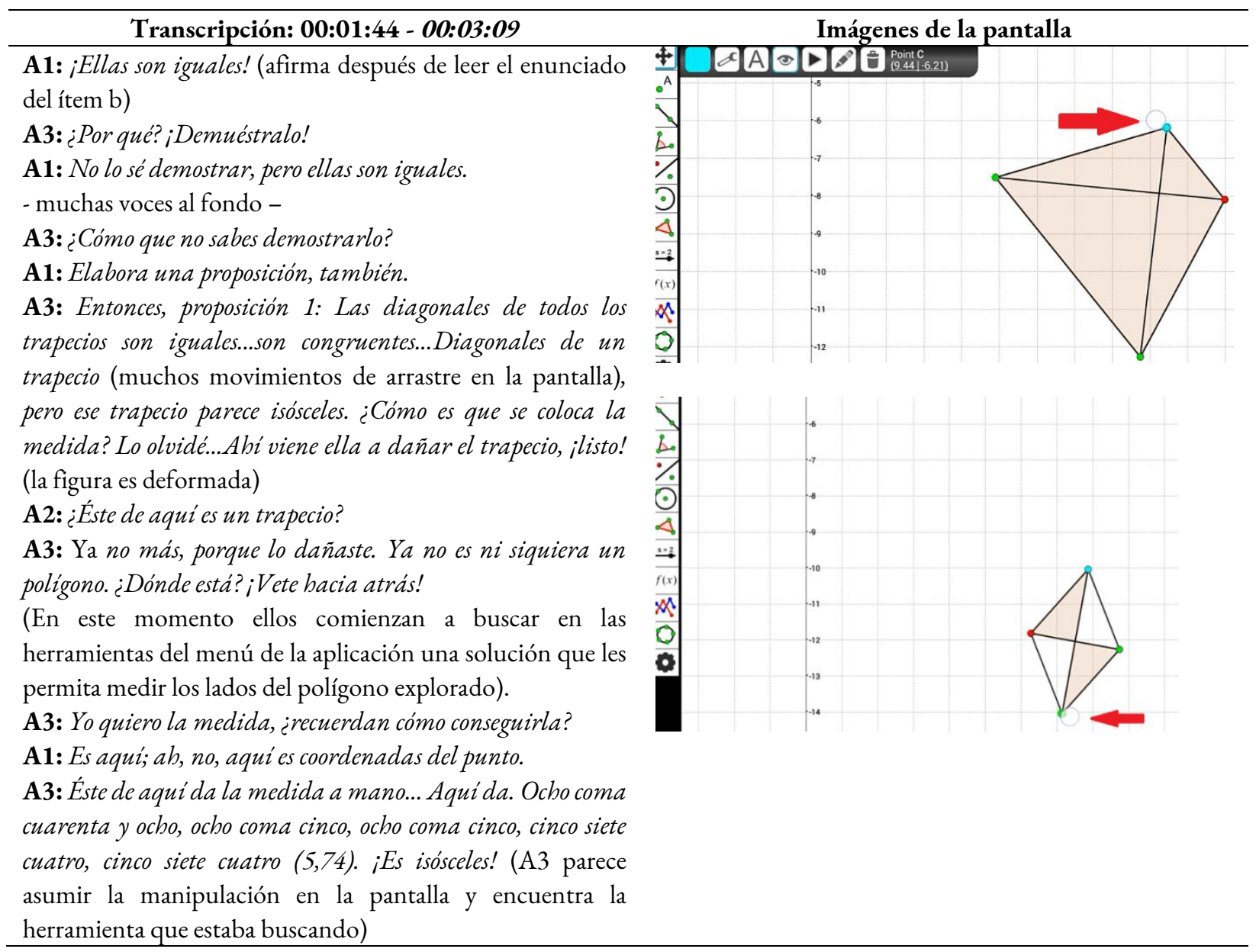

Observando el fragmento de vídeo (Tabla 9), ratificamos que el diagrama dinámico en el AGDcT en este tipo de actividad contribuyó a la exploración del polígono y a la investigación de hipótesis, ya que los alumnos tuvieron que utilizar herramientas para averiguar sus suposiciones. Podemos observar este hecho en la intervención: "A3: Éste de aqui da la medida a mano... Aqui da. Ocho coma cuarenta y ocho, ocho coma cinco, ocho coma cinco, cinco siete cuatro, cinco siete cuatro (5,74). iEs isósceles!”. Este tipo de observación, de acuerdo con Cirillo y Herbst (2012), no sería posible con lápiz y papel puesto que las 
informaciones requerirían ser dadas previamente, porque en papel no existe la posibilidad de movimiento. Además, la figura en la aplicación permitió que los alumnos observaran una clase (figura) de trapecios, y no solamente una única representación, un caso particular, un dibujo (Healy \& Hoyles, 2001).

También llamamos la atención sobre la deformación de la figura. Según Mariotti (2000), la no deformación de la figura puede garantizar geométricamente la demostración de la proposición, pues los procedimientos lógicos utilizados también necesitan ser considerados y aceptados, porque son elementos de la teoría. Por ejemplo, la construcción geométrica realizada para producir el trapecio también posee el carácter dinámico, factor que implica en las variaciones de objetos que tendremos al realizar la manipulación del arrastre. Tal como subraya Mariotti (2000), en la acción de arrastrar, el resultado es verdadero y la proposición puede ser demostrada a partir de una demostración geométrica.

Otro aspecto importante es el hecho de que los futuros profesores hayan explorado la función del hallazgo y de la explicación que la demostración puede asumir, tal como Villiers (2001) destaca. El proceso de investigación y organización de los enunciados lógicos es un momento importante de hallazgos para los sujetos (Villiers, 2001). Además, la demostración que ellos construyeron al final de la actividad justifica la proposición que elaboraron. En este sentido, percibimos que la demostración fue realizada primero, con el fin de justificar y verificar la hipótesis que ellos tenían como proposición. Veamos la intervención que confirma nuestra hipótesis: "A1: Vamos primero a elaborar la proposición. No, es mejor que demostremos primero."(Tabla 10).

\section{Tabla 10}

Diálogo del grupo 2 sobre la actividad (Parte 3) (Silva, 2017, p. 76)

Transcripción: 00:04:14 - 00: 05:18

A1: Ellas son congruentes.

A2: ¿Ellas se cortan en el medio?

A1: No. En el medio no. Sólo en el cuadrado es que se cortan en el medio.

A3: En el cuadrado y en el rombo también.

A1: Es verdad.

A3: ;Pero lo moviste de nuevo! A hora ellas ya no son iguales. (manipulaciones de arrastres en la pantalla)

A1: Sí lo son, amigo. De la forma cómo ella construyó, ellas son siempre iguales... ;Eh! No.

A3: Nolo son.

(A pesar de que los estudiantes piensan que los lados no paralelos del polígono que arrastraron poseen medidas diferentes, lo que ocurrió en realidad fue que, cuando ellos seleccionaron los lados, ellos arrastraron la figura tan sutilmente que no notaron que la medida no varió de un lado para otro, fueron ellos quienes movieron nuevamente la figura, al intentar medir)

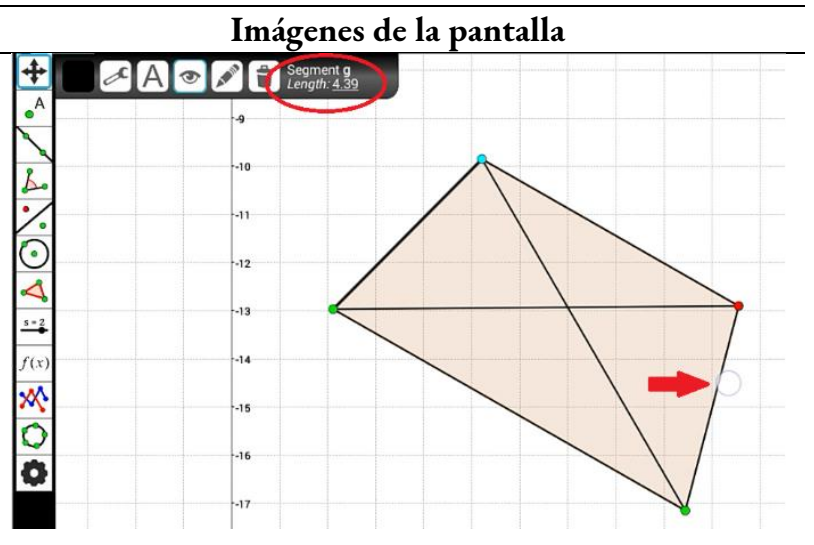




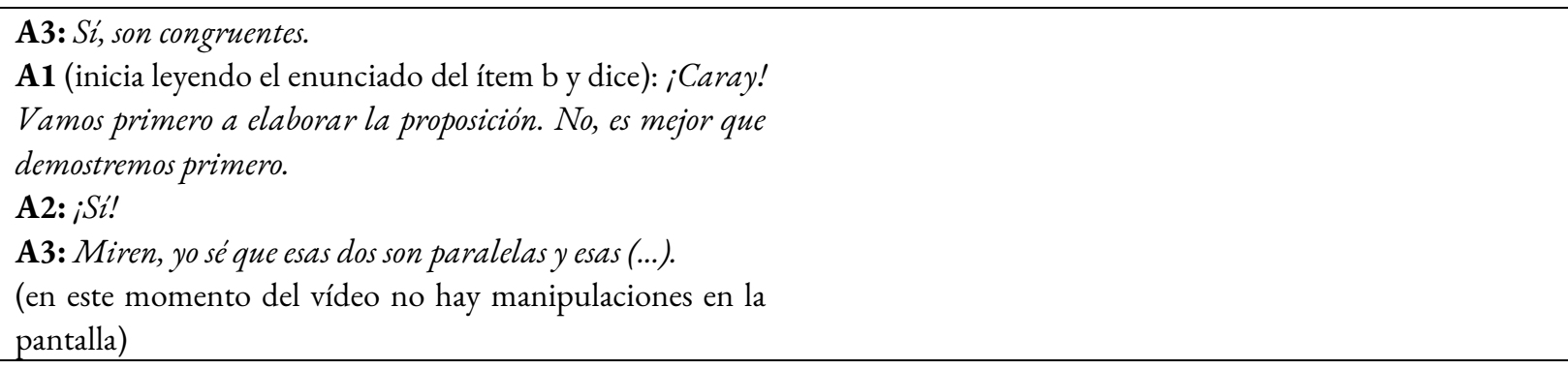

Un punto negativo que notamos en el fragmento de la Tabla 10 es la fragilidad del movimiento en la construcción, debido a la sensibilidad del dispositivo al toque en la pantalla, cuando son realizadas manipulaciones muy sutiles, al punto de confundir las suposiciones de los estudiantes, tal como se verifica en las siguientes intervenciones: "A1: Sílo son, amigo. De la forma cómo ella construyó, ellas son siempre iguales... ¡Eh! No”. “A3: No lo son.”. Además de ello, la característica del aplicativo ${ }^{18}$ de no seleccionar a través del toque directo en el objeto que se desea es un aspecto de reclamo recurrente en las implementaciones.

Con respecto a la construcción de la argumentación en AGDcT, resumimos en la Tabla 11 discursos que ilustran el razonamiento de los alumnos y su desarrollo cognitivo.

\section{Tabla 11}

Sintesis de la argumentación del grupo 2(Silva, 2017, p. 77)

\begin{tabular}{l|l}
\hline \multicolumn{1}{c}{ Extractos de la transcripción } & Manipulación (dominios) \\
\hline A1: Miren, puede ser un rectángulo... iÉse es un rectángulo? & Relacional \\
A3: No. Es un trapecio. & \\
A1: ¿Siempre va a ser un trapecio? \\
A3: Sí. \\
A1: Entiendo. Entonces, isiempre será un trapecio! (...) \\
A1: iEllas son iguales! \\
A3: ¿Por qué? ¡Demuéstralo! \\
A1: No lo sé demostrar, pero ellas son iguales. \\
A3: Entonces, proposición 1: Las diagonales de todos los trapecios son \\
iguales...son congruentes...Diagonales de un trapecio (...) \\
A3: Éste de aqui da la medida a mano... Aqui da. Ocho coma cuarenta \\
yocho, ocho coma cinco, ocho coma cinco, cinco siete cuatro, cinco siete cuatro \\
(5,74). iEs isósceles! \\
A1: Son congruentes. \\
A3: Sí, son congruentes (...) \\
A1: Vamos primero a elaborar la proposición. No, es mejor que \\
demostremos primero. \\
A2: iSi! \\
A3: Miren, yo sé que esas dos son paralelas y esas (...).
\end{tabular}

${ }^{18} \mathrm{Al}$ momento de leer este artículo, recomendamos al lector que relativice esta afirmación, debido a que el desempeño del dispositivo ya podría haber sido actualizado. Una pequeña actualización puede derivar en un gran cambio en el propósito de una tarea. 
En la Tabla 11, resulta evidente la importancia del diálogo del grupo, en conjunto con la exploración del AGDcT, en la construcción del razonamiento colectivo y en la elaboración de la demostración matemática. Las palabras en negrita indican, de forma resumida, el camino recorrido por el grupo. Expresiones como "puede ser un”, “Ése es ...?” nos indican las primeras hipótesis junto a la manipulación del arrastre. Posteriormente, la frase “¿Siempre va a ser un...?” nos muestra un cambio, es decir, lo que antes era una duda comienza a ser aclarado con el incremento de conocimientos matemáticos relacionados con el tema. Los futuros profesores llegan a una conclusión, cuando comentan "Entiendo. Entonces, siempre será...". Formulan una nueva hipótesis, pautada en conocimientos ya observados en otra situación, como nos revelan sus intervenciones: "No lo sé demostrar, pero ellas son iguales", "Las diagonales de todos los trapecios son iguales". En este momento, detienen la formulación de la proposición para verificar otra hipótesis (si el trapecio era isósceles): “Éste de aqui da la medida”, “Es isósceles!” (manipulación toque simple, dominio constructivo) y concluyen su razonamiento: "Sí, son..." Retoman la elaboración de la proposición, pero en esta ocasión comienzan por la demostración, como podemos notar en "elaborar la proposición", seguida de "demostremos primero".

\section{CONSIDERACIONES FINALES}

En este artículo, de carácter sintético e integrativo de investigaciones publicadas, opté por articular aspectos teóricos y ejemplos de tres estudios, con el propósito de facilitar la comprensión de los resultados. Aunque haya seleccionado resultados de investigaciones orientadas hacia el trabajo con cuadriláteros, en el grupo de investigación que coordino hay tesis defendidas en las que se abordan otros temas, tales como multi-numeración (Marques, 2018), isometrías (Assis, 2020) y rectas paralelas cortadas por una transversal (Henrique, 2021). Igualmente hay estudios con recogida novedosa de datos con el uso de aplicaciones móviles (Bairral et al., 2021, en prensa).

En AGDcT, hay momentos en los que la construcción del conocimiento es colectiva. En realidad, muchas veces ocurre de esa manera, aunque la interacción pueda ser una observación atenta, reflejada personalmente y/o compartida con alguien. Por lo tanto, nuestro diseño de tareas está pensado para estimular la percepción matemática de los estudiantes y hacerlos recordar o descubrir propiedades de los cuadriláteros. Aún así, defendemos que un trabajo de esa naturaleza -además de no colocar la solución de los problemas del aprendizaje en los DMcTT-puede servir de inspiración a los lectores para que planifiquen actividades en AGDcT, proporcionando a los sujetos nuevas experiencias y formas de construcción del conocimiento geométrico.

A partir de las tareas planificadas y de las interacciones establecidas entre los individuos y entre éstos y el AGD en el smartphone, los alumnos fueron manipulando sus construcciones y observando regularidades, de acuerdo con cada figura. Tal como se ilustra en las Tablas 2 y 6 , las respuestas iniciales 
de los alumnos fueron estimulantes e hicieron surgir diversas ideas para luego ser justificadas. Además, expresiones como mover, desplazar y aumentar pasaron a componer un nuevo repertorio de los sujetos con AGDcT.

Si bien los resultados aquí discutidos no provienen de una investigación longitudinal, las tablas $(1,2,4,6,8,9,10$ y 11$)$ muestran cómo el proceso formativo va potenciando en los estudiantes la mejora de sus formas de desarrollar conceptos y analizar propiedades inherentes, y la mejora en los procesos de justificación y demostración. Aunque estos procesos también carezcan de enseñanza, puesto que éstos no son espontáneos, puede ser interesante estudiar, tal vez, mediante un proyecto longitudinal, cómo potenciar aún más procesos de demostración en actividades con AGDcT.

Hemos utilizado dispositivos con rendimientos distintos (FreeGeo, Geometric Constructer, GeoGebra, Sketchometry). Algunos pueden posibilitar sólo toques simples y otros toques múltiples. Estos rendimientos generan formas de movimiento o de construcción que deben ser consideradas en el diseño de las tareas. Así pues, se torna necesario contemplar esa diferencia y, por lo tanto, cada estudio debe prestar atención a la versión del dispositivo que se utilice. Además, un mismo AGD puede variar su rendimiento en un computador o en un smartphone, como suele ocurrir con el GeoGebra. No es importante que el profesor se preocupe en permanecer observando si los alumnos usan o no determinados toques, pensados en la planificación de la clase. Lo relevante es que el educador proponga tareas que potencien hallazgos, reflexiones y el desarrollo del pensamiento matemático.

La reflexión sobre la matemática que están aprendiendo también lleva a los sujetos a avances cognitivos y afectivos importantes. Todos adquieren más control sobre su aprendizaje y desarrollan criterios para supervisar su proceso. Esa capacidad de controlar y supervisar el proceso de aprendizaje causa sentimiento de realización. La dimensión afectiva es reflexiva, o sea, es el ser humano siendo afectado. En palabras de Skliar (2014, p. 214):

Educar es sentir y pensar, no sólo la propia identidad, sino también otras formas posibles de vivir y convivir. Si eso no sucediera en las escuelas, probablemente el desierto, el páramo, la sequía ocuparian todo el paisaje de los tiempos por venir.

Las tareas y la aplicación permitieron que los alumnos manipularan, expusieran y registraran sus ideas. Por lo tanto, dinámicas de trabajo como las ilustradas en este artículo pueden estimular y mejorar exploraciones, conjeturas e investigaciones en el currículo de geometría. Si prácticas como éstas fuesen realizadas a lo largo de la Educación Básica, nuestros estudiantes tendrían un pensamiento matemático más potenciado y serían ayudados, por ejemplo, en la construcción de demostraciones matemáticas (Silva, 2017). Por ello, creemos que la articulación entre tecnología digital móvil con toques en pantalla y actividades planificadas adecuadamente para su uso puede proporcionar calidad en el aprendizaje y el interés de los discentes por estudios más avanzados en matemática. 
En definitiva, el diseño de la actividad contribuyó a la constitución de demostraciones y a la producción de argumentos autorales de los futuros profesores, enriqueció la dinámica dialógica entre todos, así como posibilitó la creación y exploración de objetos geométricos diferentes de los modelos estándar y estáticos de los libros didácticos. Por lo tanto, nuestra investigación (Silva, 2017) aporta contribuciones didácticas -con la implementación de una dinámica interactiva y de análisis compartida de respuestas por el propio colectivo- y cognitivas, con la promoción y reflexión de nuevas formas de justificar y generar procesos de demostración, mediante recursos y estrategias variadas para mejorar el conocimiento de futuros profesores de matemática.

A pesar de que no hemos realizado actividades que pudieran registrar el hallazgo de las inclusiones de las clases de los cuadriláteros -por ejemplo, todo cuadrado es un rectángulo y, por consiguiente, posee todas las propiedades de éste último-, aún así consideramos valiosos los debates y cuestionamientos hechos durante las clases, ya que éstos contribuyeron a la construcción del conocimiento de los alumnos sobre diferentes aspectos de los polígonos. Comparto, aquí, una sugerencia más de desarrollo de esta investigación: analizar cómo los estudiantes establecen relación de inclusión de cuadriláteros, cuando las interacciones, la movilidad y los toques en pantalla entran en escena.

\section{ACLARATORIAS}

El autor no tiene conflicto de interés que declarar. El artículo ha sido financiado con recursos propios del autor.

\section{REFERENCIAS}

Arzarello, F., Bairral, M., \& Dané, C. (2014). Moving from dragging to touchscreen: geometrical learning with geometric dynamic software. Teaching Mathematics and its Applications, 33(1), 39-51. https://doi.org/10.1093/teamat/hru002

Arzarello, F., Bairral, M., Dané, C., \& Iijima, Y. (2013). Ways of manipulation touchscreen in one geometrical dynamic software. En E. Faggiano, \& A. Montone (Eds.), Proceeding of the $11^{\text {th }}$ International Conference on Technology in Mathematics Teaching (ICTMT11) (pp. 59-64). Universidad de Bari.

Arzarello, F., Paola, D., Robutti, O., \& Sabena, C. (2009). Gestures as semiotic resources in the mathematics classroom. Educational Studies in Mathematics. Special issue on Gestures and Multimodality in the Construction of Mathematical Meaning, 70(2), 97-109. https://doi.org/10.1007/s10649-008-9163-z 
Assis, A. R. de (2020). Alunos do ensino médio realizando toques em telas e aplicando isometrias com GeoGebra [tese de doutorado não disponível online, Universidade Federal Rural do Rio de Janeiro].

Assis, A., \& Bairral, M. (2019). Using touchscreen devices to improve plane transformation in high school classroom. International Journal for Research in Mathematics Education (RIPEM), 9(1), 45-60. http://sbem.iuri0094.hospedagemdesites.ws/revista/index.php/ripem/article/view/2078

Bairral, M. (2013, 29 de septiembre-2 de octubre). Do clique ao touchscreen: novas formas de interação e de aprendizado matemático [paper presentation]. 36a Reunião Anual da Anped, Goiânia. http://36reuniao.anped.org.br/pdfs trabalhos aprovados/gt19 trabalhos pdfs/gt19 2867 tex to.pdf

Bairral, M. A. (2017). As manipulações em tela compondo a dimensão corporificada da cognição matemática. Jornal Internacional de Estudos em Educação Matemática (JIEEM), 10(2), 104-111. https://doi.org/10.17921/2176-5634.2017v10n2p104-111

Bairral, M. (2019). Touching on screen, exploring and making sense regarding quadrilaterals with FreeGeo App. Quaderni di Ricerca in Didattica: Matematica (Special number 7), 421-425.

Bairral, M. (2020). Not only what is written counts! Touchscreen enhancing our cognition and language. Global Journal of Human-Social Science (G), 20(5), 1-10. https://doi.org/10.34257/GJHSSGVOL20IS5PG1

Bairral, M. A., Arzarello, F., \& Assis, A. (2015). High School students rotating shapes in GeoGebra with touchscreen. Quaderni di Ricerca in Didattica: Matematica, 25(suplemento 2). Proceedings CIEAEM 67, 103-108. http://math.unipa.it/ grim/CIEAEM\%2067 Proceedings QRDM Issue\%2025 Suppl.2.pdf

Bairral, M., Arzarello, F., \& Assis, A. (2017). Domains of manipulation in touchscreen devices and some didactic, cognitive and epistemological implications for improving geometric thinking. En G. Aldon, F. Hitt, L. Bazzini, \& U. Gellert (Eds.), Mathematics and technology: a C.I.E.A.E.M source book (pp. 113-142). Springer. https://doi.org/10.1007/978-3-319-51380-5 7

Bairral, M. A., Assis, A., \& Silva, B. C. (2016). Mãos em ação em dispositivos touchscreen na Educação Matemática. Edur UFRRJ.

Bairral, M. A., \& Barreira, J. C. F. (2017). Algumas particularidades de ambientes de geometria dinâmica na educação geométrica. Revista do Instituto GeoGebra de São Paulo, 6(2), 46-64. https://revistas.pucsp.br/index.php/IGISP/article/view/35378 
Bairral, M., \& Carvalho, M. (Eds.). (2019). Dispositivos móveis no ensino de matemática: tablets छ smartphones. Editora Livraria da Física.

Bairral, M. A., \& Henrique, M. P. (Eds.). (2021). Smartphones com toques da Educação Matemática: mãos que pensam, inovam, ensinam, aprendem e pesquisam. CRV. https://doi.org/10.24824/978655578728.3

Bairral, M., Henrique, M. P., \& Assis, A. (2021, en prensa). Moving parallel and transversal lines with touches on smartphones: a look through screenrecording. The Mathematics Enthusiast, 19.

Bairral, M. A., \& Powell, A. (2015). Identificação e análise de objetos e relações em Virtual Math Teams. En A. Powell (Ed.), Métodos de pesquisa em Educação Matemática: usando escrita, video e internet (pp. 127-150). Mercado de Letras.

Bairral, M. A., \& Silva, E. R. de C. (2018). Trabalhando quadriláteros em smartphones: alunos de uma escola pública descobrindo e produzindo propriedades. Debates em Educação, 10(22), 164-190. https://doi.org/10.28998/2175-6600.2018v10n22p164-190

Bussi, M. G. B., \& Mariotti, M. A. (2008). Semiotic mediation in the mathematics classroom: artifacts and signs after a Vygotskian perspective. En L. D. English, M. B. Bussi, G. A. Jones, R. A. Lesh, B. Sriraman, \& D. Tirosh (Eds.), Handbook of International Research in Mathematics Education (second revised edition, pp. 746-783). Routledge / Taylor \& Francis Group.

Calder, N., Larkin, K., \& Sinclair, N. (Eds.). (2018). Using mobile technologies in the teaching and learning of Mathematics. Springer. https://doi.org/10.1007/978-3-319-90179-4

Chao, T., Murray, E., \& Star, J. (2016). Helping mathematics teachers develop noticing skills: utilizing smartphone technology for one- on-one teacher/student interviews. Contemporary Issues in Technology and Teacher Education, 16(1), 22-37. https://citejournal.org/wp-content/uploads/2016/05/v16i1math1.pdf

Cirillo, M., \& Herbst, P. (2012). Moving toward more authentic proof practices in geometry. The Mathematics Educator, 21(2), 11-33. https://128.192.239.209/tme/article/view/1964/1869

Dalcín, M., \& Molfino, V. (2012). Clasificación particional de cuadriláteros como fuente de demostraciones y construcciones en la formación inicial de profesores. Revista do Instituto GeoGebra de São Paulo, 1(1), LXXXI-XCVII. https://revistas.pucsp.br/index.php/IGISP/article/view/8841 
Healy, L., \& Hoyles, C. (2001). Software tools for geometrical problem solving: potentials and pitfalls. International Journal of Computers for Mathematical Learning, 6(3), 235-256.

http://doi.org/10.1023/A:1013305627916

Henrique, M. P. (2021). Metáforas e toques em tela: potencializando aprendizagens discentes no estudo de retas paralelas e transversais [tese de doutorado não disponível online, Universidade Federal Rural do Rio de Janeiro].

Laborde, C. (2001). Integration of technology in the design of geometry tasks with cabri geometry. International Journal of Computers for Mathematical Learning, 6(3), 283-217. https://doi.org/10.1023/A:1013309728825

Lasa, A., \& Wilhelmi, M. R. (2013). Use of GeoGebra in explorative, illustrative and demonstrative moments. Revista do Instituto GeoGebra de São Paulo, 2(1), 52-64. https://revistas.pucsp.br/index.php/IGISP/article/view/15160

Mariotti, M. A. (2000). Introduction to proof: the mediation of a dynamic software environment. Educational Studies in Mathematics, 44(1-3), 25-53. https://doi.org/10.1023/A:1012733122556

Mariotti, M. A. (2019). A geometria em sala de aula: reflexóes sobre ensino e aprendizagem (S. de S. Melo, Trans.). Editora UFPE.

Marques, W. (2018). Multinumeramentos em smartphones de alunos do ensino médio sob telas da neurociência [tese de doutorado não disponível online, Universidade Federal Rural do Rio de Janeiro].

$\mathrm{Ng}$, O. (2016). Comparing calculus communication across static and dynamic environments using a multimodal approach. Digital Experiences in Mathematics Education, 2(2), 115-141. https://doi.org/10.1007/s40751-016-0014-8

Ng, O., \& Sinclair, N. (2015). “Area without numbers”: using touchscreen dynamic geometry to reason about shape. Canadian Journal of Science, Mathematics and Technology Education, 15(1), 84-101. https://doi.org/10.1080/14926156.2014.993048

Park, D., Lee, J., \& Kim, S. (2011). Investigating the affective quality of interactivity by motion feedback in mobile touchscreen user interfaces. International Journal of Human-Computer Studies, 69(12), 839-853. https://doi.org/10.1016/j.ijhcs.2011.06.006

Silva, B. C. C. de (2017). Justificativas e argumentaçóes no aprendizado de quadriláteros: uma intervenção com papel, lápis e dispositivos móveis [dissertação de mestrado, Universidade Federal 
Rural do Rio de Janeiro]. Biblioteca Digital de Teses e Dissertações da UFRRJ. https://tede.ufrrj.br/jspui/handle/jspui/4409

Silva, B. C. C. da, \& Bairral, M. (2019). Justificativas e argumentações no aprendizado de quadriláteros com o FreeGeo. En M. Bairral, \& M. Carvalho (Eds.), Dispositivos móveis no ensino de matemática: tablets e smartphones (pp. 159-178). Editora Livraria da Física.

Sinclair, N., \& Freitas, E. de (2014). The haptic nature of gesture: rethinking gesture with new multitouch digital technologies. Gesture, 14(3), 351-374.

https://doi.org/10.1075/gest.14.3.04sin

Sinclair, N., \& Robutti, O. (2013). Technology and the role of proof: the case of dynamic geometry. En M. A. K. Clements, A. J. Bishop, C. Keitel, J. Kilpatrick, \& F. K. S. Leung (Eds.), Third International Handbook of Mathematics Education (pp. 571-596). Springer. https://doi.org/10.1007/978-1-4614-4684-2_19

Sinclair, N., \& Yurita, V. (2008). To be or to become: how dynamic geometry changes discourse. Research in Mathematics Education, 10(2), 135-150. https://doi.org/10.1080/14794800802233670

Skliar, C. (2014). Desobedecer a linguagem: educar. Autêntica.

Villiers, M. de (1994). The role and function of a hierarchical classification of quadrilaterals. For The learning of Mathematics, (14), 11-18. https://flm-journal.org/Articles/58360C6934555B2AC78983AE5FE21.pdf

Villiers, M. D. de (2001). Papel e funções da demonstração no trabalho com o Sketchpad. Educação e Matemática, (62), 33-36.

\section{Cómo citar este artículo:}

Bairral, M. (2021). Interacciones, toques en pantalla y aprendizaje de cuadriláteros. Revista Venezolana de Investigación en Educación Matemática (REVIEM), 1(2), e202107. https://doi.org/10.54541/reviem.v1i2.9 


\section{ANEXOS $^{19}$}

Anexo I: Ficha de sondeo inicial

Nombre:

$\operatorname{Edad}^{20}$ :

1) ¿Cuáles son los nombres de los cuadriláteros que tienen:
a) ¿Un único par de lados paralelos?
b) ¿Dos pares de lados paralelos?
c) ¿Cuatro ángulos rectos?
d) ¿Cuatro lados de igual medida?
e) ¿Un único par de lados opuestos de igual medida?
f) ¿Dos pares de lados de igual medida?
g) ¿Sólo un par de ángulos rectos?
h) ¿Dos ángulos agudos y dos obtusos?
i) ¿Un único ángulo agudo y un único ángulo obtuso?

2) Sólo una de las siguientes frases es falsa. ¿Cuál?
a) El cuadrado es un paralelogramo.
b) El rectángulo es un paralelogramo.
c) El trapecio es un paralelogramo.
d) El rombo es un paralelogramo.
e) Todo cuadrilátero tiene cuatro lados, cuatro vértices y cuatro ángulos.

\footnotetext{
${ }^{19}$ Los Anexos I, II, III, IV, V y VI son tomados de Bairral y Silva (2018). Por su parte, el Anexo VII es tomado de Arzarello $e t$ al. (2013). Finalmente, el Anexo VIII es tomado de Assis (2020).

${ }^{20}$ Aunque la edad de cada alumno pueda ser informada en la primera ficha, optamos por dejarla como una posible información a ser obtenida en cada encuentro.
} 
Anexo II: Ficha de actividad 1

Nombre:

Edad:

1) Construye un cuadrado en el FreeGeo sin usar la herramienta "polígono regular" y sin la ayuda de la cuadrícula:

a) Mueve libremente el cuadrado y realiza dos observaciones que consideres interesante.

b) ¿Es posible transformar tu cuadrado en un rectángulo? ¿Qué hace diferente a un cuadrado de un rectángulo?

c) Construye un rectángulo. 
Anexo III: Ficha de actividad 2

Nombre:

Edad:

1) Observa las figuras abajo.

a) Identifica cuadrados en las siguientes figuras:

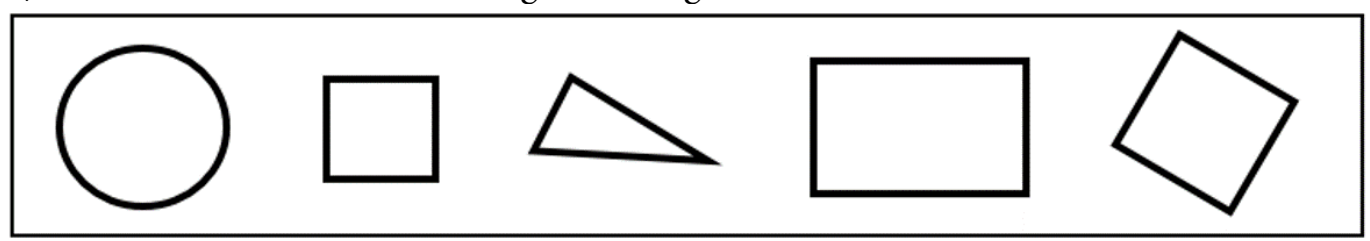

b) Identifica rectángulos en las siguientes figuras:

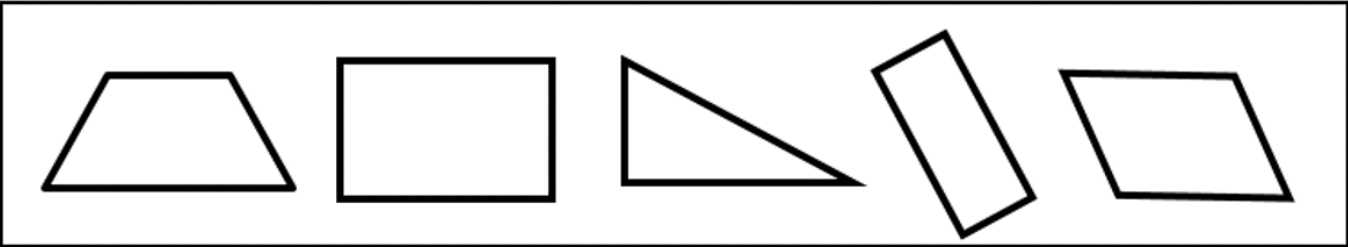

c) Identifica rombos en las siguientes figuras:

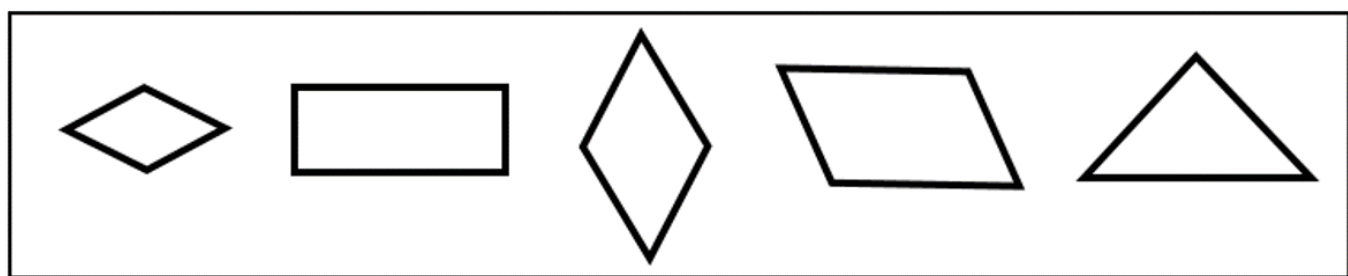

2) Construye en el FreeGeo un rombo y realiza tres observaciones: sobre sus lados, ángulos y diagonales. 
Anexo IV: Ficha de actividad 3

Nombre:

Edad:

1) Construye el siguiente cuadrilátero especial, siguiendo las instrucciones abajo:

a) Construye una recta $\mathrm{AB}$.

b) Construye un punto $C$ que no pertenezca a la recta $A B$.

c) Construye una recta que pase por los puntos $\mathrm{B}$ y $\mathrm{C}$.

d) Construye una recta paralela a $\mathrm{AB}$ que pase por el punto $\mathrm{C}$.

e) Construye una recta paralela a $\mathrm{BC}$ que pase por el punto $\mathrm{A}$.

f) Construye un punto $\mathrm{D}$ que sea intersección de las últimas dos rectas que construiste.

g) Construye un polígono que tenga como vértices los puntos $\mathrm{A}, \mathrm{B}, \mathrm{C}$ y D.

h) Haz clic sobre cada una de las rectas auxiliares y las ocultas.

2) Para mostrar la medida de los lados del cuadrilátero, construye los segmentos $\mathrm{AB}, \mathrm{BC}, \mathrm{CD}$ y $\mathrm{DA}$. Luego, muestra el valor de estos segmentos. Desplaza los vértices del cuadrilátero e investiga las medidas de los lados. ¿Qué observas?

3) Para mostrar la medida de los ángulos internos del cuadrilátero, selecciona Ángulo y haz clic sobre los vértices en sentido horario. Desplaza los vértices del cuadrilátero e investiga las medidas de los ángulos. ¿Qué observas?

4) El cuadrilátero que investigaste se llama paralelogramo. ¿Por qué crees que esta figura tiene ese nombre?

5) ¿Cuáles son las características de un paralelogramo? 
Anexo V: Ficha de actividad 4

Nombre:

Edad:

1) Construye el siguiente cuadrilátero especial, siguiendo las instrucciones abajo:

a) Construye una recta $\mathrm{AB}$.

b) Construye un punto $C$ que no pertenezca a la recta $A B$.

c) Construye una recta paralela a la recta $\mathrm{AB}$ y que pase por el punto $\mathrm{C}$.

d) Construye un punto $\mathrm{D}$ sobre la recta que pase por el punto $\mathrm{C}$, tal como se ilustra en la figura abajo:

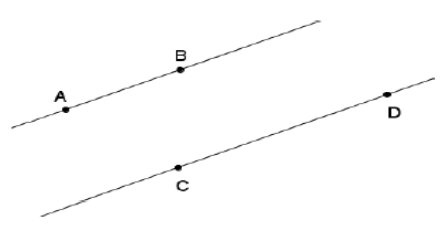

e) Construye un polígono que tenga como vértices los puntos $\mathrm{A}, \mathrm{B}, \mathrm{D}$ y C.

2) ¿Cuántos pares de lados paralelos posee ese cuadrilátero?

3) Para mostrar la medida de los lados del cuadrilátero, construye los segmentos AB, BD, DC y CA. Luego, muestra el valor de esos segmentos. Desplaza los vértices del cuadrilátero e investiga las medidas de los lados. ¿Qué observas?

4) Para mostrar la medida de los ángulos internos del cuadrilátero, selecciona ángulo y haz clic sobre los vértices en sentido horario. Desplaza los vértices del cuadrilátero e investiga las medidas de los ángulos. ¿Qué observas?

5) El cuadrilátero que investigaste se llama trapecio. ¿Cuáles son las características de este cuadrilátero?

6) Desplaza los vértices hasta que los dos lados no paralelos del trapecio tengan la misma medida. Ese trapecio es llamado trapecio isósceles. Haz un esbozo de la figura obtenida en el espacio abajo, indicando las medidas de los ángulos y de los lados.

7) ¿Qué observas en relación a las medidas de los ángulos del trapecio rectángulo? 
Anexo VI: Ficha de sondeo final

Nombre:

Edad:

1) Diga cuál(es) son los cuadriláteros (cuadrado, rectángulo, trapecio, rombo, paralelogramo) que poseen:

a) un único par de lados paralelos.

b) dos pares de lados paralelos.

c) cuatro ángulos rectos.

d) cuatro lados de igual medida.

e) un único par de lados opuestos de igual medida.

f) dos pares de lados de igual medida.

g) sólo un par de ángulos rectos.

h) dos ángulos agudos y dos obtusos.

i) un único ángulo agudo y un único ángulo obtuso.

2) Sólo una de las siguientes frases es falsa. ¿Cuál?

a) El cuadrado es un paralelogramo.

b) El rectángulo es un paralelogramo.

c) El trapecio es un paralelogramo.

d) El rombo es un paralelogramo.

e) Todo cuadrilátero tiene cuatro lados, cuatro vértices y cuatro ángulos.

La frase marcada anteriormente es falsa porque:

3) Los rectángulos y los cuadrados tienen una misma propiedad. ¿Cuál?

4) Los rombos y los cuadrados tienen una misma propiedad. ¿Cuál?

5) ¿Cuál es la diferencia entre los trapecios y los paralelogramos?

6) Una lista mínima de propiedades de una figura contiene las propiedades suficientes y necesarias para definirla. Por ejemplo, una lista mínima para un cuadrado garantizará que la figura sea un cuadrado, y, si cualquier propiedad fuese retirada de esa lista, la figura dejará de ser un cuadrado. Intercambia ideas con tus compañeros de grupo y escribe la lista mínima de propiedades que definen a cada tipo de cuadrilátero.

\begin{tabular}{|l|l|}
\hline Cuadrado & \\
\hline Rectángulo & \\
\hline Rombo & \\
\hline Paralelogramo & \\
\hline Trapecio & \\
\hline
\end{tabular}




\section{Anexo VII}

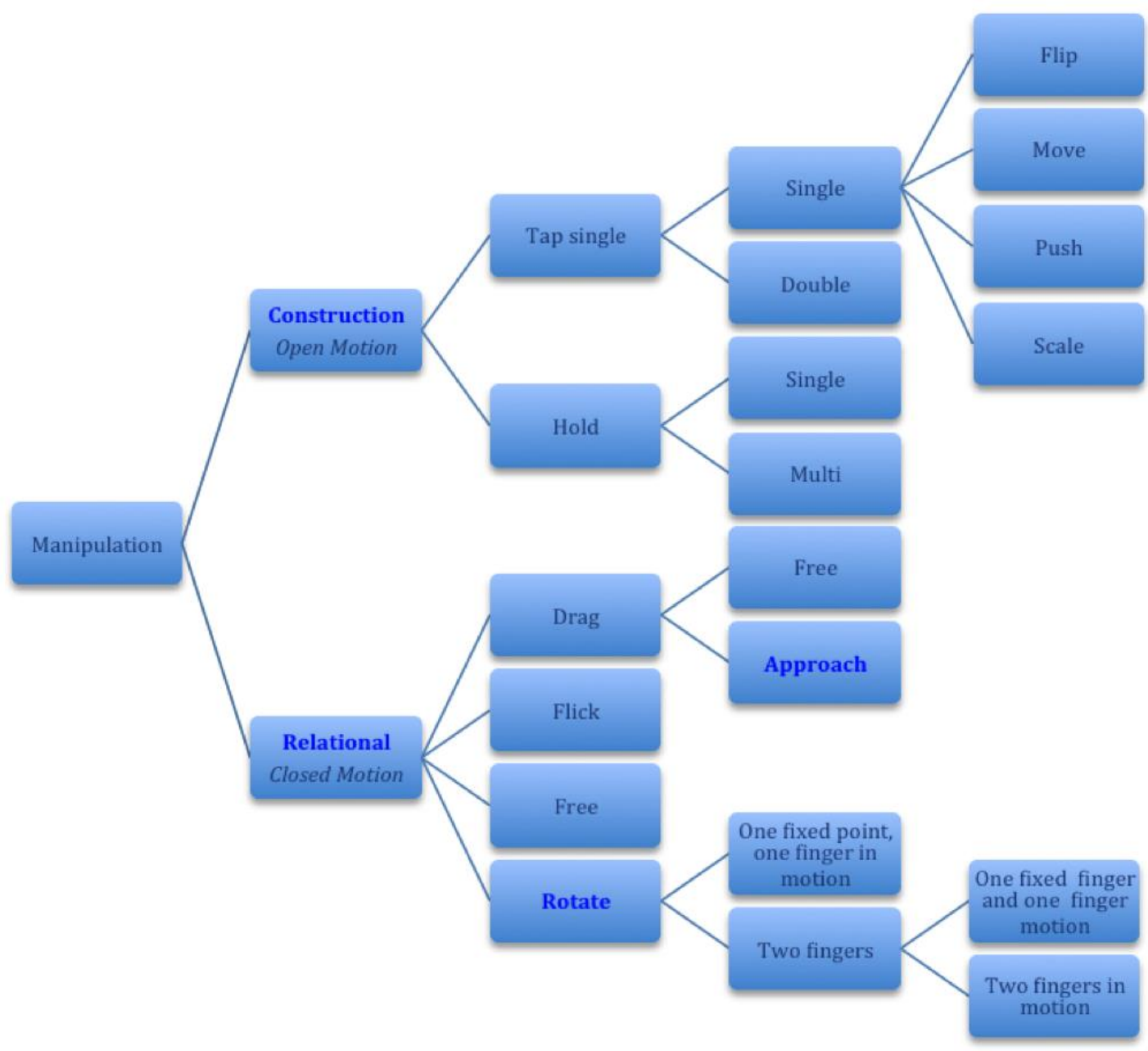




\section{Anexo VIII}

\begin{tabular}{|c|c|c|c|c|}
\hline \multicolumn{3}{|c|}{ Manipulación } & Toques & Acción \\
\hline \multicolumn{3}{|c|}{ 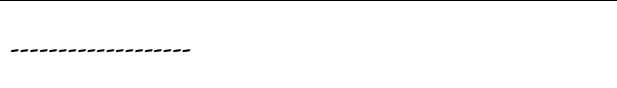 } & & $\begin{array}{l}\text { Pausa, análisis, reflexión, } \\
\text { observación, negociación }\end{array}$ \\
\hline \multirow{3}{*}{ Toque } & \multicolumn{2}{|l|}{ Simple } & & $\begin{array}{l}\text { Selecciona algún elemento de la } \\
\text { construcción o toque sobre la área } \\
\text { de trabajo }\end{array}$ \\
\hline & \multicolumn{2}{|c|}{ Simple y fijo } & & $\begin{array}{l}\text { Selecciona algún elemento y con } \\
\text { momento para analizar }\end{array}$ \\
\hline & \multicolumn{2}{|l|}{ Doble } & & $\begin{array}{l}\text { Selecciona alguna herramienta } \\
\text { oculta (pero no mantiene fijo) }\end{array}$ \\
\hline \multirow{6}{*}{ Arrastre } & \multicolumn{2}{|l|}{ para arriba } & & $\begin{array}{l}\text { Selecciona un elemento o } \\
\text { construcción y arrastra para arriba }\end{array}$ \\
\hline & \multicolumn{2}{|l|}{ para abajo } & & $\begin{array}{l}\text { Selecciona un elemento o } \\
\text { construcción y arrastra para abajo }\end{array}$ \\
\hline & \multicolumn{2}{|c|}{ para la izquierda } & & $\begin{array}{l}\text { Selecciona un elemento o } \\
\text { construcción y arrastra para la } \\
\text { izquierda }\end{array}$ \\
\hline & \multicolumn{2}{|c|}{ para la derecha } & & $\begin{array}{l}\text { Selecciona un elemento o } \\
\text { construcción y arrastra para la } \\
\text { derecha }\end{array}$ \\
\hline & \multicolumn{2}{|l|}{ Ampliar } & & $\begin{array}{l}\text { Zoom hacia afuera para visualizar } \\
\text { mejor la construcción }\end{array}$ \\
\hline & \multicolumn{2}{|l|}{ Reducción } & & $\begin{array}{l}\text { Zoom hacia adentro para visualizar } \\
\text { mejor el área de construcción, la } \\
\text { propia construcción como un todo } \\
\text { u otros elementos }\end{array}$ \\
\hline \multirow{7}{*}{ Rotación } & \multirow{3}{*}{\multicolumn{2}{|c|}{ Con un dedo }} & & Rotación libre \\
\hline & & & & Rota para la izquierda \\
\hline & & & & Rota para la derecha \\
\hline & \multirow{4}{*}{ Dos dedos } & \multirow{2}{*}{$\begin{array}{l}\text { Uno fijo y } \\
\text { el otro en } \\
\text { movimient } \\
\text { o }\end{array}$} & & $\begin{array}{l}\text { Fija un dedo y rota para la derecha } \\
\text { con el otro }\end{array}$ \\
\hline & & & & $\begin{array}{l}\text { Fija un dedo y rota para la izquierda } \\
\text { con el otro }\end{array}$ \\
\hline & & \multirow{2}{*}{$\begin{array}{l}\text { Los dos en } \\
\text { movimient } \\
\text { o }\end{array}$} & & $\begin{array}{l}\text { Selecciona utilizando dos dedos y } \\
\text { rota para la izquierda }\end{array}$ \\
\hline & & & & $\begin{array}{l}\text { Selecciona utilizando dos dedos y } \\
\text { rota para la derecha }\end{array}$ \\
\hline
\end{tabular}




\section{Marcelo Bairral}

c) (7)

Copyright $@$ (2021. Marcelo Bairral. Esta obra está protegida por una licencia Creative Commons 4.0. International (CC BY 4.0).

Usted es libre para Compartir — copiar y redistribuir el material en cualquier medio o formato - y Adaptar el documento - remezclar, transformar y crear a partir del material — para cualquier propósito, incluso para fines comerciales, siempre que cumpla la condición de:

Atribución: Usted debe dar crédito a la obra original de manera adecuada, proporcionar un enlace a la licencia, e indicar si se han realizado cambios. Puede hacerlo en cualquier forma razonable, pero no de forma tal que sugiera que tiene el apoyo del licenciante o lo recibe por el uso que hace de la obra.

$\underline{\text { Resumen de licencia - Texto completo de la licencia }}$ 